\title{
C-FGF23 peptide alleviates hypoferremia during acute inflammation
}

\begin{abstract}
Rafiou Agoro, ${ }^{1}$ Min Young Park, ${ }^{1}$ Carole Le Henaff, ${ }^{1}$ Stanislovas Jankauskas, ${ }^{1}$ Alina Gaias, ${ }^{1}$ Gaozhi Chen, ${ }^{2}$ Moosa Mohammadi ${ }^{3}$ and Despina Sitara ${ }^{1,4}$

${ }^{1}$ Department of Basic Science and Craniofacial Biology, NYU College of Dentistry, New York, NY, USA; ${ }^{2}$ Chemical Biology Research Center, School of Pharmaceutical Sciences, Wenzhou Medical University, Wenzhou, China; ${ }^{3}$ Departments of Biochemistry and Molecular Pharmacology, NYU School of Medicine, New York, NY, USA and ${ }^{4}$ Department of Medicine, NYU School of Medicine, New York, NY, USA
\end{abstract}

\section{ABSTRACT}

$\mathrm{H}$ ypoferremia results as an acute phase response to infection and inflammation aiming to reduce iron availability to pathogens. Activation of toll-like receptors (TLR), the key sensors of the innate immune system, induces hypoferremia mainly through the rise of the iron hormone hepcidin. Conversely, stimulation of erythropoiesis suppresses hepcidin expression via induction of the erythropoietin-responsive hormone erythroferrone. Iron deficiency stimulates transcription of the osteocyte-secreted protein FGF23. Here we hypothesized that induction of FGF23 in response to TLR4 activation is a potent contributor to hypoferremia and, thus, impairment of its activity may alleviate hypoferremia induced by lipopolysaccharide (LPS), a TLR 4 agonist. We used the C-terminal tail of FGF23 to impair endogenous full-length FGF23 signaling in wildtype mice, and investigated its impact on hypoferremia. Our data show that FGF23 is induced as early as pro-inflammatory cytokines in response to LPS, followed by upregulation of hepcidin and downregulation of erythropoietin (Epo) expression in addition to decreased serum iron and transferrin saturation. Further, LPS-induced hepatic and circulating hepcidin were significantly reduced by FGF23 signaling disruption. Accordingly, iron sequestration in liver and spleen caused by TLR4 activation was completely abrogated by FGF23 signaling inhibition, resulting in alleviation of serum iron and transferrin saturation deficit. Taken together, our studies highlight for the first time that inhibition of FGF23 signaling alleviates LPS-induced acute hypoferremia.

\section{Introduction}

Hypoferremia develops as a response to innate immune system activation following inflammation or infection. Iron is an essential element required for the survival, proliferation, and virulence of pathogens. The hypoferremic response is an important defense mechanism that aims to prevent iron bioavailability to pathogens by sequestration of iron within macrophages, resulting in a significant reduction in circulating iron levels. ${ }^{1-3}$ Toll-like receptors (TLR) are key components of the innate immune system that recognize pathogen-associated molecular patterns (PAMP), and are responsible for sensing invading pathogens. Activation of TLR signaling plays a pivotal role in the development of the hypoferremic host response.,3 Lipopolysaccharide (LPS), a PAMP that is the major component of the outer membrane of Gram-negative bacteria, is known to activate TLR4. Injection of LPS to mice or humans causes a rapid release of pro-inflammatory cytokines and triggers a well characterized acute phase inflammatory response followed by an induction of the hepatic hormone hepcidin, the central regulator of iron homeostasis. ${ }^{4.6}$

Hepcidin binds to ferroportin, the only known iron exporter, and causes its internalization and degradation, ${ }^{7}$ resulting in iron sequestration in reticulo-endothelial macrophages, hepatocytes, and duodenal enterocytes, and subsequently decreased circulating iron levels. ${ }^{8}$ This situation of circulating iron restriction promotes ineffective erythropoiesis and results in anemia of inflammation, a form of anemia commonly associated with infectious diseases, cancer, and chronic kidney disease (CKD). ${ }^{9}$
Ferrata Storti Foundation

Haematologica 2021

Volume 106(2):391-403

\section{Correspondence:}

DESPINA SITARA

ds199@nyu.edu

Received: September 2, 2019.

Accepted: March 13, 2020.

Pre-published: March 19, 2020.

https://doi.org/10.3324/haematol.2019.237040

(C)2021 Ferrata Storti Foundation

Material published in Haematologica is covered by copyright. All rights are reserved to the Ferrata Storti Foundation. Use of published material is allowed under the following terms and conditions:

https://creativecommons.org/licenses/by-nc/4.0/legalcode. Copies of published material are allowed for personal or internal use. Sharing published material for non-commercial purposes is subject to the following conditions: https://creativecommons.org/licenses/by-nc/4.0/legalcode, sect. 3. Reproducing and sharing published material for com mercial purposes is not allowed without permission in writing from the publisher. 
CKD patients have increased iron losses due to chronic bleeding from impaired intestinal iron absorption, uremiaassociated platelet dysfunction, frequent phlebotomy, and blood trapping in the dialysis apparatus. ${ }^{10}$ In addition, circulating levels of fibroblast growth factor 23 (FGF23) dramatically increase in $\mathrm{CKD}$, reaching up to 2,000 -fold above the normal range in advanced renal failure. ${ }^{11}$ Recent studies have associated iron deficiency with the increase in FGF23 levels. ${ }^{12,13}$

FGF23 has been identified as the gene responsible for the phosphate wasting disorder autosomal dominant hypophosphatemic rickets (ADHR) ${ }^{14}$ and as a causative factor of tumor-induced osteomalacia. ${ }^{15}$ FGF23 is a bonederived endocrine regulator of phosphate and vitamin $\mathrm{D}$ homeostasis and bone mineralization. ${ }^{16-20}$ FGF23 inhibits phosphate reabsorption in the renal proximal tubule by suppressing the expression of the type II sodium-phosphate transporters $\mathrm{NaPi} 2 \mathrm{a}$ and $\mathrm{NaPi} 2 \mathrm{c}$ in the brush border membrane. ${ }^{17,21-23}$ Furthermore, FGF23 reduces circulating levels of 1,25-dihydroxyvitamin $\mathrm{D}_{3}$ by suppressing the renal expression of $1 \alpha$-hydroxylase, the enzyme required for production of 1,25-dihydroxyvitamin D3, and increasing the activity of 24-hydroxylase, the enzyme that metabolizes 1,25 -dihydroxyvitamin D3. ${ }^{17}$ FGF23 requires a co-receptor, klotho, for FGF receptor (FGFR) activation, and its effects are mediated through multiple FGFR, with FGFR1 being the principal receptor to mediate the effects of FGF23 on phosphate regulation, and FGFR3 and 4 mediating the FGF23 effects on vitamin D metabolism. ${ }^{24}$ In the kidney, FGF23 binds to the FGFR-klotho complex and induces signaling through Ras/MAPK, PIBK/Akt, and PLC $\gamma /$ PKC downstream pathways which regulate mineral metabolism genes. ${ }^{25}$ FGF23 activity is regulated post-translationally by proteolytic cleavage between $\operatorname{Arg}^{179}$ and $\mathrm{Ser}^{180}$, and O-linked glycosylation. Cleavage of the intact full-length FGF23 peptide by a subtilisin-like pro-protein convertase abolishes its biologic activity. Intact FGF23 contains an arginine-X-X-arginine ( $R X X R$ ) motif which is recognized by pro-protein convertases such as furin. This motif is located at the boundary between the FGF core homology domain, which interacts with the FGFR, and the 72-residue-long C-terminal tail of FGF23, which interacts with the co-receptor Klotho. ${ }^{26-28}$

We recently reported that inhibition of FGF23 signaling rescues renal anemia in a mouse model of $\mathrm{CKD} .{ }^{29}$ Here, we investigated the effect of inhibition of FGF23 signaling in a mouse model of LPS-induced hypoferremia. Our results show that FGF23 is induced in response to LPS prior to hepcidin upregulation followed by downregulation of Epo expression. Moreover, we show that inhibition of FGF23 signaling alleviates hypoferremia and attenuates dysregulation of erythropoiesis in a mouse model of acute inflammation induced by LPS.

\section{Methods}

\section{Animals}

C57BL/6 wild-type (WT) male mice between 8 and 10 weeks of age were purchased from The Jackson Laboratory (Bar Harbor, ME, USA) and housed at the New York University (NYU) College of Dentistry Animal Facility, where they were kept on a light/dark (12 hours [h]/12h) cycle at $23^{\circ} \mathrm{C}$, and received food (standard lab chow) containing 185 parts per million iron (Teklad 2018S; Harlan) and water ad libitum. All animal protocols were approved by the
Institutional Animal Care and Use Committee (IACUC) at NYU. Mice were treated with a single intraperitoneal (i.p.) injection of 1 $\mathrm{mg} / \mathrm{kg}$ of an FGF23 blocking peptide (provided by Dr. M. Mohammadi, NYU School of Medicine) or vehicle (HEPESbuffered saline; $25 \mathrm{mM}$ HEPES-NaOH pH7.5, $150 \mathrm{mM} \mathrm{NaCl}$ ). The FGF23 blocking peptide is the 72 aa long C-terminal tail of human FGF23 (residues Ser180-Ile251), which corresponds to the C-terminal fragment of FGF23 generated by proteolytic cleavage at the RXXR motif. ${ }^{27}$ Eight hours post treatment, acute inflammation was induced by a single i.p injection of $50 \mu \mathrm{g} / \mathrm{kg}$ of LPS (E. coli 055:B5, Sigma-Aldrich, St Louis, MO, USA). An equal volume of sterile saline solution $(0.9 \% \mathrm{NaCl})$ was used as vehicle control. Mice were euthanized $4 \mathrm{~h}$ after LPS treatment. In longitudinal studies, saline- and LPS-treated mice were euthanized after 1, 2, 4, 6,12 , or 24 h of LPS treatment.

\section{Blood, tissue, and serum collection}

Mice were immediately necropsied after euthanasia and blood was collected by cardiac puncture. Complete blood count was performed using the VetScan HM5 Hematology Analyzer (ABAXIS, Union City, CA, USA). Serum was obtained after blood centrifugation at 3,500 rpm for 10 minutes (min). Liver, spleen, kidney, bones, and bone marrow cells obtained from femora and tibiae, were collected, snap-frozen in liquid nitrogen, and stored at $-80^{\circ} \mathrm{C}$ until further use.

\section{Serum measurements}

Serum FGF23 levels were measured using the mouse FGF23 Intact and C-terminal ELISA assays (Quidel Corporation/Immutopics International, San Clemente, CA, USA). The intact assay detects exclusively biologically intact FGF23 (iFGF23), whereas the C-terminus (cFGF23) assay is capable of detecting both the intact molecule and its C-terminal fragments. Serum phosphorus was determined by colorimetric measurements using the Stanbio Phosphorus Liqui-UV Test reagent (Stanbio Laboratory, Boerne, TX, USA). Serum iron and transferrin saturation were measured using the Iron-TIBC kit from Pointe Scientific (Canton, MI, USA). Serum hepcidin was measured using the Hepcidin Murine-Compete ELISA kit (Intrinsic LifeSciences, La Jolla, CA, USA). Serum EPO levels were measured using the Rat/Mouse EPO Quantikine ELISA kit (R\&D Systems, Minneapolis, MN, USA).

\section{Tissue iron measurement}

Hepatic and splenic iron concentration was measured by the Ferrozine colorimetric method, as described elsewhere. ${ }^{30,31}$

\section{RNA isolation, reverse transcription, and real-time quantitative polymerase chain reaction analysis}

Total RNA was extracted from kidneys, liver, whole bone, spleen, and bone marrow using Trizol (Ambion; Life Technologies, Carlsbad, CA, USA) according to the manufacturer's protocol (Molecular Research Center, Cincinnati, OH, USA). Synthesis of cDNA was performed using the High Capacity cDNA Reverse Transcription Kit as described by the manufacturer (Applied Biosystems; Thermo Fisher Scientific, Waltham, MA, USA). cDNA was amplified by quantitative polymerase chain reaction (qPCR) using the PerfecCTa SYBR Green SuperMix (Quanta Biosciences, Gaithersburg, MD, USA). All primers used in this study are listed in the Online Supplementary Table S1. mRNA levels were normalized to the housekeeping gene (Gadph or Hprt) in the same cDNA sample. The relative transcript expression of a gene is given as $\Delta C t=C t_{\text {target }}-C t_{\text {reference }}$. The fold change in gene expression, as compared to control mice, was determined as $2^{-\Delta \Delta \mathrm{Ct}}$ values $\left(\Delta \Delta C \mathrm{t}=\Delta C \mathrm{t}_{\text {treated }}-\Delta C \mathrm{t}_{\text {control }}\right)$. 
A

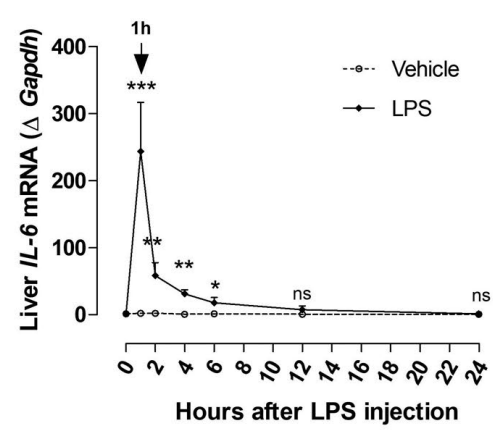

B

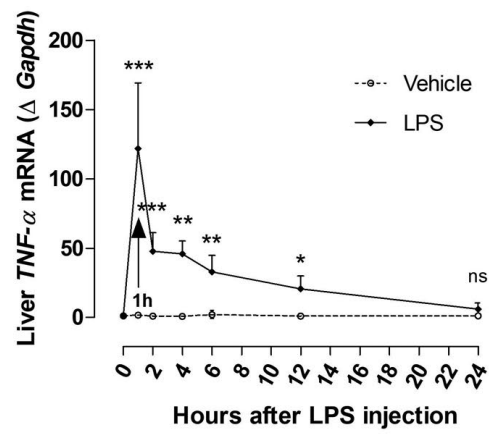

C

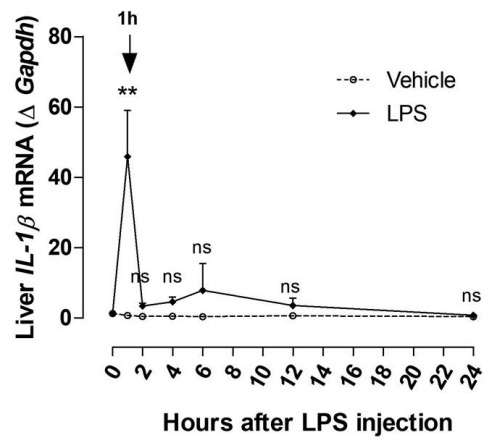

D

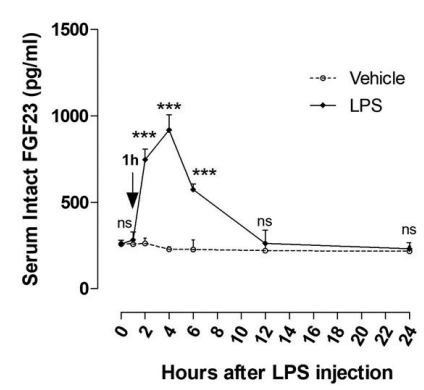

$\mathrm{E}$

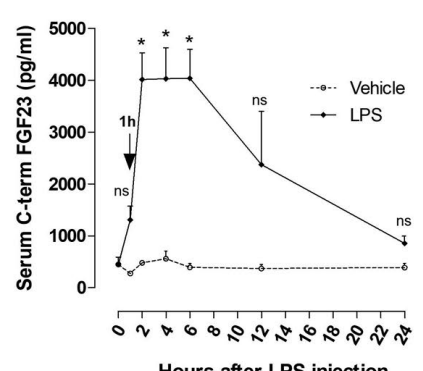

F

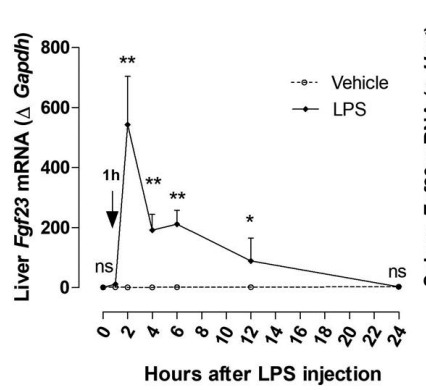

G

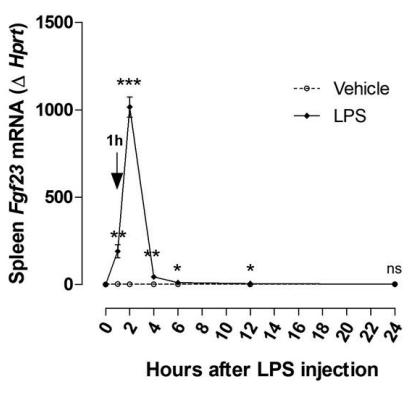

Figure 1. Early induction of Fgr23 in response to lipopolysaccharide (LPS). C57BL/6J mice were injected intraperitoneally (i.p.) with a single dose of saline (0.9\% $\mathrm{NaCl}$, indicated as Vehicle) or LPS (50 $\mathrm{gg} / \mathrm{kg}$ ). Samples were collected at 0 (saline injection only), 1, 2, 4, 6, 12, and 24 hours (h) after treatment and total mRNA was isolated. (A-C) Quantitative real-time polymerase chain reaction (qRT-PCR) of (A) IL-6, (B) TNF- $\alpha$, and (C) IL-1 $\beta$ expression in liver. (D and E) Serum concentration of (D) intact and (E) C-terminal FGF23 measured by ELISA. (F and G) qRT-PCR for Fgf23 expression in (F) liver and (G) spleen. Data are expressed as fold change $\left(2^{-\Delta \Delta C t}\right)$ relative to housekeeping genes Gapdh or Hprt. Samples were measured in duplicates (vehicle, $n=3-4 ;$ LPS, $n=5-8$ ), and data are represented as mean+standard deviation. All data were analyzed for normality with Shapiro-Wilk test and equivalence of variance using Levene's test. For samples with normal distribution, two-way ANOVA was performed in each vehicle- or LPS-treated group compared to $0 \mathrm{~h}$ with Bonferroni's multiple comparison test (D). When the samples were not in normal distribution and equivalence of variance, data were analyzed with non-parametric Kruskal-Wallis test (A-C, E-G). ns: not significant, $* P<0.05$, $* \star P<0.01$, $* * * P<0.001$ compared to $0 \mathrm{~h}$.

\section{Western blotting}

Snap frozen samples of spleen and liver were homogenized in an ice-cold RIPA buffer (Sigma-Aldrich, St. Louis, MO, USA) with addition of EDTA-free HaltTM Protease Inhibitor Single-use Cocktail and HaltTM Phosphatase Inhibitor Single-Use Cocktail (both from Thermo Scientific, Rockford, IL, USA) and were lysed for $2 \mathrm{~h}$ on ice with vortexing every $15 \mathrm{~min}$. After lysis, samples were centrifuged for $20 \mathrm{~min}$ at 13,000 g and protein concentration was measured in supernatants by Quick Start Bradford Dye Reagent according to the manufacturer's instructions. For each sample, $30 \mathrm{mg}$ of protein was loaded into $4-20 \%$ Mini-Protean ${ }^{\circledR}$ TGX $^{\mathrm{TM}}$ Precast Protein Gels. Electrophoresis was performed for 18 min on a constant voltage of $300 \mathrm{~V}$ in a Tris/Glycine/SDS buffer. Then proteins were transferred to $0.45 \mu \mathrm{m}$ nitrocellulose membrane by wet method with Tris/Glycine Transfer buffer (Quality Biological Inc., Gaithersburg, MD, USA) with the addition of $20 \%$ methanol (Fisher Scientific, Hampton, NH, USA) for $1 \mathrm{~h}$ on a constant voltage of $110 \mathrm{~V}$. Membranes were then washed with Trisbuffered saline Tween (TBST) (Fisher Scientific, Hampton, NH, USA) and blocked for $2 \mathrm{~h}$ at room temperature (RT) with either $5 \%$ non-fat dry milk (LabScientific Inc., Highlands, NJ, USA) in TBST (for anti-ferroportin and anti-STAT3 antibodies) or 5\% BSA (Sigma-Aldrich, St. Louis, MO, USA) in TBST (for anti-phosphoSTAT3 antibodies) and washed four times with TBST. Membranes were stained with primary antibodies overnight at $4^{\circ} \mathrm{C}$ : anti-STAT3 mouse antibody $(1: 1,000$ on $5 \%$ non-fat dry milk-TBST), anti-phospho-STAT3 (Tyr705) rabbit antibody
(1:2,000 on 5\% BSA-TBST) (\#9139 and \#9145, respectively, Cell Signaling Technology, Danvers, MA, USA), anti-ferroportin rabbit antibody (1:1,000 on $5 \%$ non-fat dry milk TBST) (\#MTP11-S, Alpha Diagnostic Intl Inc., San Antonio, TX, USA), and anti-Actin goat antibody (1:1,000 on TBST) (sc-1616, Santa Cruz Biotechnology Inc., Dallas, TX, USA). Membranes were stained with secondary antibodies diluted 1:10,000 on TBST for $30 \mathrm{~min}$ at RT: mouse-anti-rabbit IgG-HRP, goat-anti-mouse IgG-HRP and Rb-anti-Gt IgG-HRP (all from Santa Cruz Biotechnology Inc., Dallas, TX, USA). After incubation, membranes were washed with TBST four times. Visualization was performed with Clarity Western ECL Substrate and ChemiDocTM XRS+ imager, images were analyzed with Image $\mathrm{Lab}^{\mathrm{TM}}$, and protein signal intensity was calculated with ImageJ (NIH, Bethesda, MD, USA). All reagents, materials, devices and software were purchased from Bio-Rad Laboratories Inc., Hercules, CA, USA, unless otherwise specified.

\section{Statistical analysis}

All data were analyzed for normal distribution by Shapiro-Wilk tests and "Normal Q-Q Plot" graphs. If normal distribution was not achieved, data were aligned in rank transformation and a normality test was performed. The equality of variance was determined with Levene's test. If both normal distribution and equality of variance were demonstrated, data were analyzed by two-way analysis of variance (ANOVA) with Bonferroni's multiple comparison test. When data were not normally distributed, non-parametric test was performed by Kruskal-Wallis test using SigmaStat soft- 
A

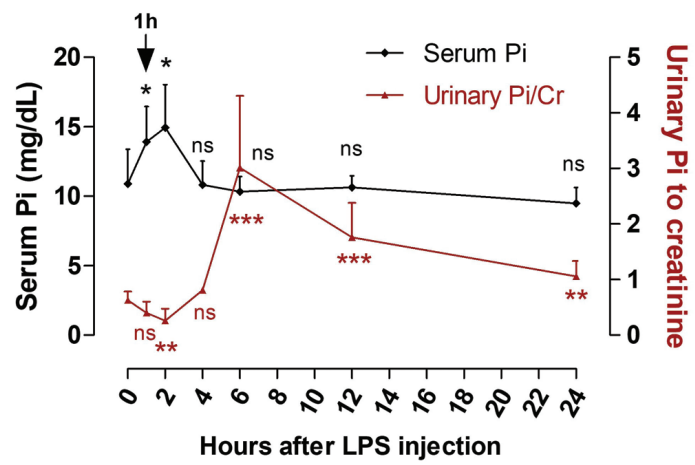

C

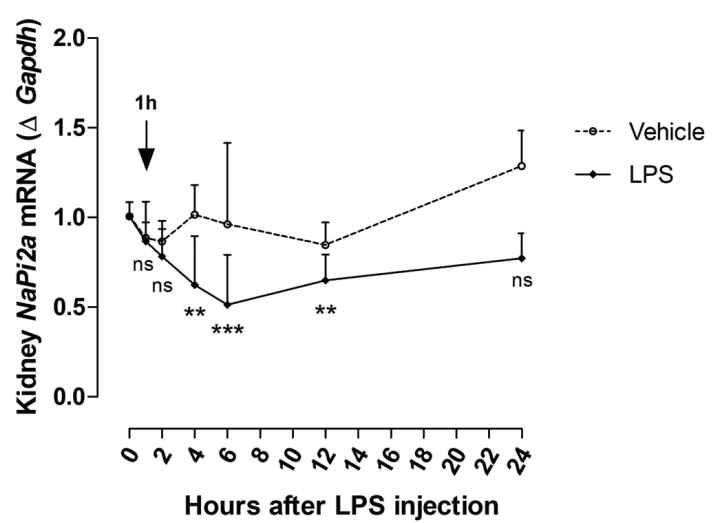

B

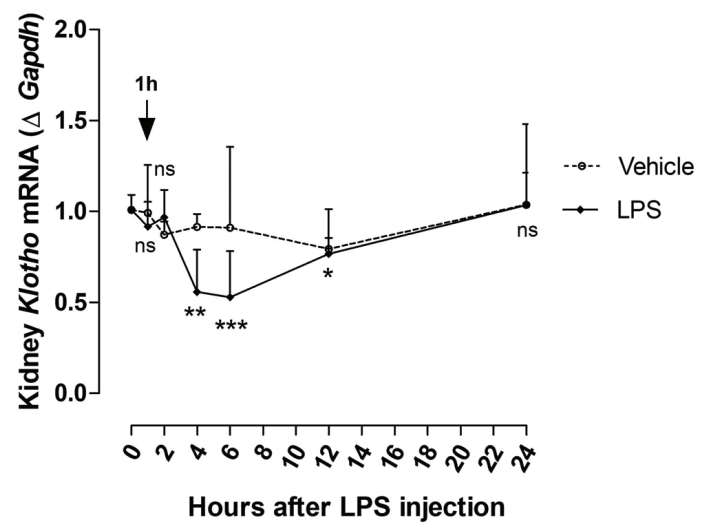

D

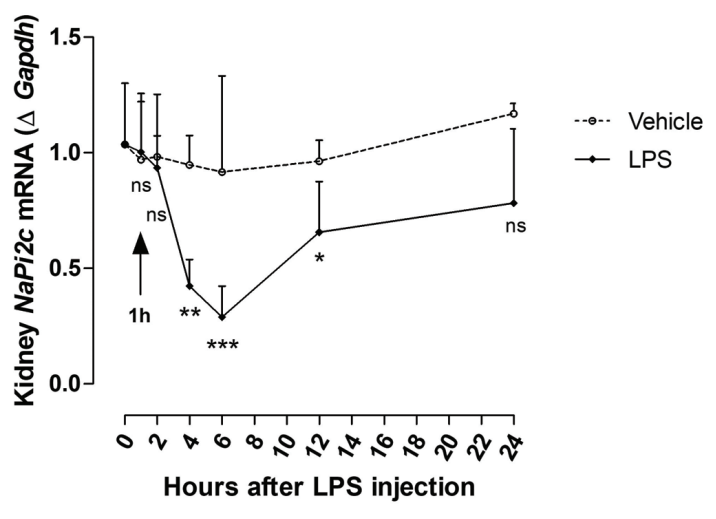

Figure 2. Regulation of phosphate homeostasis following lipopolysaccharide (LPS) administration. (A) Phosphate levels measured in serum and urine at 0 , 1, 2, 4, 6, 12, and 24 hours (h) after i.p. injection of LPS (50 $\mu \mathrm{g} / \mathrm{kg}$ ). (B-D) Quantitative real-time polymerase chain reaction (qRT-PCR) for renal (B) Klotho, (C) NaPi2a, and (D) NaPi2c expression. Data are expressed as fold change $\left(2^{-\Delta \Delta c t}\right)$ relative to housekeeping gene Gapdh. Samples were measured in duplicates (vehicle, $n=3-4$; LPS, $\mathrm{n}=5-8)$. Data are represented as mean+standard deviation. All data were analyzed for normality with Shapiro-Wilk test and equivalence of variance using Levene's test. For serum Pi, data were analyzed by non-parametric Kruskal-Wallis test; for the ratio of urinary Pi to creatinine, data were aligned in RANK transformation and analyzed with one-way ANOVA followed by Bonferroni's multiple comparison test (A). With the samples showing normal distribution, two-way ANOVA was performed in each vehicle- or LPS-treated group compared to $\mathrm{O} \mathrm{h}$ with Bonferroni's multiple comparison test $(\mathrm{C})$. The samples not in normal distribution were analyzed with nonparametric Kruskal-Wallis test (B and D). ns: not significant, $* P<0.05, * * P<0.01, * * * P<0.001$ compared to 0 h.

ware (SPSS Sciences, Chicago, IL, USA) and GraphPad Prism 8 (2019 GraphPad Software, Inc. La Jolla, CA, USA). All values are presented as mean+standard deviation (SD). $P<0.05$ was considered statistically significant.

\section{Results}

\section{Early induction of Fgf23 and regulation of phosphate homeostasis in response to lipopolysaccharide}

To identify the series of events that take place in response to inflammation, we carried out longitudinal studies during which we injected a sub-lethal dose (50 $\mu \mathrm{g} / \mathrm{kg}$ ) of LPS to wild-type mice and evaluated its effect on inflammatory markers, FGF23 levels, and phosphate homeostasis after 1, 2, 4, 6, 12, and $24 \mathrm{~h}$. As expected, liver pro-inflammatory cytokines such as $I L-6, T N F-\alpha$, and $I L-1 \beta$ were significantly induced during the first $6 \mathrm{~h}$ of LPS treatment, reaching a peak at $1 \mathrm{~h}$, and returning progressively to baseline levels by $12 \mathrm{~h}$ upon resolution of inflammation (Figure 1A-C). Furthermore, we investigated the effect of LPS treatment on Fgf23 expression and circulating FGF23 levels. Both serum intact and Cterminal FGF23 levels were significantly elevated between 2-6 h or 2-12 h, respectively, in response to LPS (Figure 1D and E). Bone Fgf23 expression increased $4 \mathrm{~h}$ after LPS treatment (Online Supplementary Figure S1A). However, in liver, Fgf23 expression was significantly upregulated $2 \mathrm{~h}$ after LPS treatment and remained elevated up to $12 \mathrm{~h}$, before progressively returning to basal levels by $24 \mathrm{~h}$ (Figure 1F). In addition, Fgf23 expression was markedly increased in spleen and bone marrow during the first $12 \mathrm{~h}$ after LPS treatment and returned to basal levels by $24 \mathrm{~h}$ (Figure $1 \mathrm{G}$ and Online Supplementary Figure $S 1 B)$. Serum phosphate levels were significantly increased the first $2 \mathrm{~h}$ of LPS treatment, and they were associated with a concurrent significant decrease in urinary phosphate excretion (Figure 2A), possibly causing the induction of FGF23. In a counter-regulatory manner, the increase in circulating FGF23 led to a significant increase in urinary phosphate levels $6 \mathrm{~h}$ after LPS treatment, resulting in normalization of serum phosphate levels (Figure 2A). In kidney, FGF23 binds to the FGFRKlotho complex to induce phosphate excretion by downregulating the sodium-phosphate transporters $\mathrm{NaPi} 2 \mathrm{a} / \mathrm{c}$. Consistent with increased FGF23 levels and urinary phosphate excretion, renal expression of NaPi2a, NaPi2c, as well as Klotho, decreased significantly during the period of FGF23 elevation in response to LPS, before returning to basal levels by $24 \mathrm{~h}$ (Figure 2B-D). Taken together, our data demonstrate that, in response to LPS, FGF23 is induced as early as inflammatory markers, resulting in 
Table 1. Effects of lipopolysaccharide (LPS) and fibroblast growth factor 23 (FGF23) blocking peptide on hematologic parameters in mice.

\begin{tabular}{lcccc} 
& Ct & LPS & FGF23 BL & FEF23 BL+ LPS \\
WBC, $\times 10^{9} / \mathrm{L}$ & $2.47 \pm 0.41$ & $2.79 \pm 0.78$ & $2.16 \pm 0.80$ & $1.72 \pm 0.76^{\mathrm{b}^{* *}}$ \\
Lymphocyte, $\times 10^{9} / \mathrm{L}$ & $1.73 \pm 0.66$ & $1.22 \pm 0.30$ & $2.09 \pm 0.53$ & $0.80 \pm 0.36^{\mathrm{b}^{*}, \mathrm{c}^{* * *}}$ \\
\hline Neutrophil, $\times 10^{9} / \mathrm{L}$ & $0.19 \pm 0.22$ & $1.49 \pm 0.53^{\mathrm{a}^{* * *}}$ & $0.23 \pm 0.22$ & $0.81 \pm 0.45^{\mathrm{b}^{* *}, \mathrm{c}^{*}}$ \\
RBC, $\times 10^{12} / \mathrm{L}$ & $9.80 \pm 0.35$ & $10.85 \pm 0.59^{\mathrm{a}^{* *}}$ & $10.09 \pm 0.22$ & $10.28 \pm 0.6 \mathrm{~b}^{\mathrm{b}^{*}}$ \\
$\mathrm{Hb}, \mathrm{g} / \mathrm{dL}$ & $14.18 \pm 0.41$ & $15.23 \pm 0.94^{\mathrm{a}^{*}}$ & $14.62 \pm 0.69$ & $14.45 \pm 0.6 \mathrm{~b}^{\mathrm{b}^{*}}$ \\
Hematocrit, \% & $45.24 \pm 1.55$ & $48.96 \pm 2.49^{\mathrm{a}^{* *}}$ & $46.96 \pm 1.12$ & $46.32 \pm 2.2 \mathrm{~b}^{\mathrm{b}^{*}}$ \\
\hline RDW, \% & $18.14 \pm 1.18$ & $17.41 \pm 0.43$ & $17.50 \pm 0.37$ & $16.73 \pm 0.50^{\mathrm{b}^{* *}, \mathrm{c}^{*}}$ \\
MCV, fL & $46.00 \pm 0.00$ & $45.60 \pm 1.65$ & $46.40 \pm 0.55$ & $45.20 \pm 1.48$
\end{tabular}

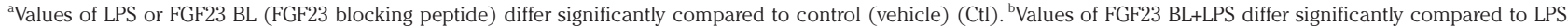
${ }^{c}$ Values of FGF23 BL+LPS differ significantly compared to FGF23 BL. ${ }^{*} P<0.05,{ }^{*} P<0.01,{ }^{* *} P<0.001$

regulation of phosphate homeostasis by promoting phosphate excretion.

\section{Acute inflammation induces hypoferremia and downregulates Epo expression}

Inflammation is a major stimulus for hepcidin synthesis in the liver. Specifically, treatment of hepatocytes with LPS leads to increase in hepcidin levels. ${ }^{32,33}$ In agreement with published studies, our data show that hepcidin expression started rising $1 \mathrm{~h}$ after LPS treatment with significant upregulation between 4-6 h after LPS (Figure $3 \mathrm{~A}$ ), consistent with the presence of inflammation (Figure $1 \mathrm{~A}-\mathrm{C}$ ), and returned to basal levels after resolution of inflammation (Figure 3A). The increase in hepcidin resulted in progressive and significant reduction in circulating iron and transferrin saturation levels (Figure $3 \mathrm{~B}$ and $\mathrm{C}$ ), followed by recovery from hypoferremia once inflammation was resolved and hepcidin levels returned to normal. This drop in serum iron levels was accompanied by a significant decrease in ferroportin expression in liver and spleen (Figure 3D and E), leading to iron retention in these organs (Figure 3F and Online Supplementary Figure S2) and increased spleen weight (Figure $3 \mathrm{G}$ ). In addition, expression of the acute phase protein lipocalin $2(\operatorname{Lcn} 2)$, which plays a role as an iron sequester, ${ }^{34}$ was increased after $4 \mathrm{~h}$ of LPS and remained elevated up to $24 \mathrm{~h}$ post LPS treatment (Figure $3 \mathrm{H}$ ). These data demonstrate that LPS induces hypoferremia associated with downregulation of ferroportin and iron retention in liver and spleen.

Studies have shown that pro-inflammatory cytokines, such as IL-6, TNF- $\alpha$, and IL-1 $\beta$, increased by inflammation and/or infection, inhibit renal erythropoietin (Epo) production, the primary regulator of erythropoiesis, and therefore suppress erythropoiesis. 35.37 This is known as anemia of inflammation. Specifically, it has been shown that administration of LPS in rats significantly suppressed renal Epo gene expression. ${ }^{36}$ In our studies, we found that renal Epo expression was significantly downregulated after 4-6 h (Figure 3I).

\section{Inhibition of FGF23 signaling alleviates hypoferremia induced by acute inflammation}

It has been reported that the C-terminal tail of FGF23 mediates the binding of FGF23 to the FGFR-Klotho complex. ${ }^{27,38}$ Studies have shown that the 72 -aa-long C-terminal tail of FGF23 (residues Ser180-Ile251), which corresponds to the C-terminal fragment of FGF23 generated by proteolytic cleavage at the RXXR motif, impairs FGF23 signaling and activity by acting as endogenous inhibitor of the full-length FGF23 for binding to the FGFR-Klotho complex. ${ }^{27} \mathrm{We}$ previously reported that inhibition of FGF23 signaling using this FGF23 blocking peptide (BL) ameliorates iron deficiency in a mouse model of $\mathrm{CKD}{ }^{29}$ Since FGF23 significantly increases in response to LPS, here we assessed the effect of inhibiting FGF23 signaling in a mouse model of hypoferremia induced by acute inflammation. Based on the results of our longitudinal studies, we opted to assess the effect of FGF23 signaling inhibition $4 \mathrm{~h}$ post LPS treatment. At this time point, we observed maximum induction of FGF23 and hepcidin, associated with a significant decrease in serum iron, transferrin saturation, and erythropoietin, during upregulation of pro-inflammatory cytokines.

Our data show that the increase in FGF23 in response to LPS was significantly reduced after inhibition of FGF23 signaling (Figure 4A-C). Administration of LPS triggered an acute inflammatory response (Figure 5A-C) that led to induction of hepcidin (Figure 5D and E). However, blocking FGF23 signaling significantly decreased hepatic expression of $T N F-\alpha$ without affecting $I L-6$ and $I L-1 \beta$ mRNA expression in LPS-treated mice (Figure 5A-C). Importantly, the increase in hepatic and circulating hepcidin levels induced by LPS was significantly reduced when FGF23 signaling was impaired (Figure $5 \mathrm{D}$ and $\mathrm{E}$ ). We also found that inhibition of FGF23 signaling did not impact phosphorylation of STAT3, the main intracellular regulator of hepcidin induction by IL6, as treatment with cFGF23 did not affect LPS-induced upregulation of hepatic STAT3 phosphorylation (Online Supplementary Figure S3). Therefore, suppression of hepcidin by cFGF23 appears to be independent of the IL6/STAT3 pathway. These data suggest that inhibiting FGF23 signaling reduces LPS-induced hepcidin in mice with normal kidney function.

Increased hepcidin leads to iron sequestration in tissues and subsequent decrease in circulating iron levels. ${ }^{7,8,39}$ As expected, LPS elicited hypoferremia after $4 \mathrm{~h}$ with a significant decrease in serum iron and transferrin saturation (Figure 6A and B), associated with a robust reduction in liver and spleen ferroportin $(F p n)$ mRNA (Figure 6C and D). Downregulation of ferroportin resulted in increased hepatic and splenic iron content (Figure $6 \mathrm{E}$ and F), which correlated with increased hepatic expression of the iron storage protein ferritin $\mathrm{H}(F t h)$ (Figure 6G) and the iron sequester lipocalin 2 (Lcn2) (Figure $6 \mathrm{H}$ ). The number of circulating neutrophils, which are the main source of lipocalin secretion, was 
A

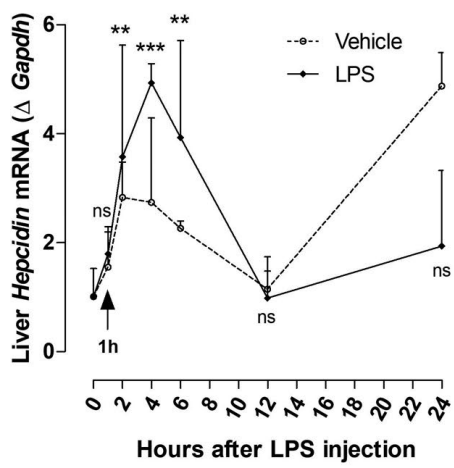

D

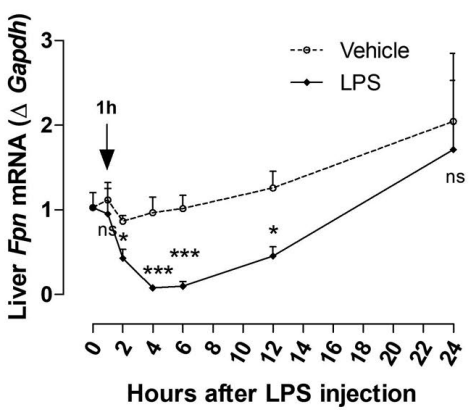

G

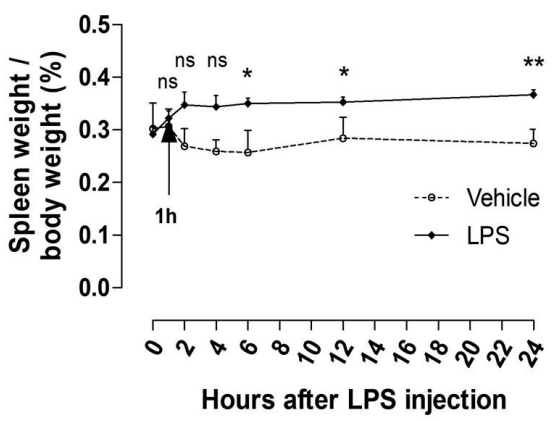

B

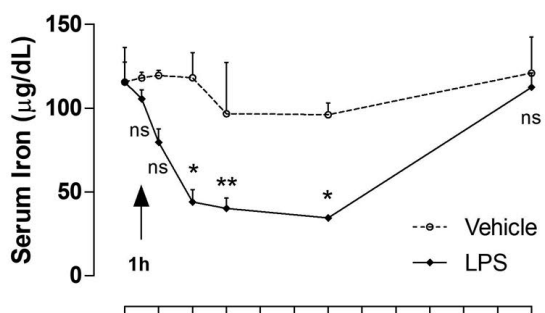

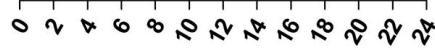

Hours after LPS injection

E

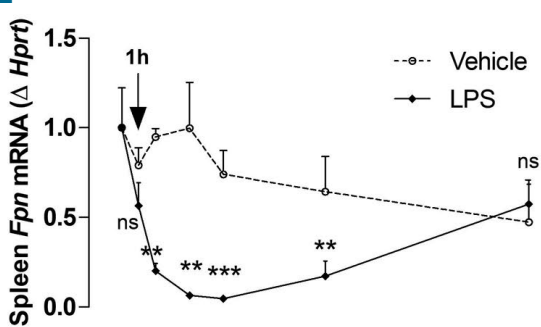

$0 \eta * 68,1^{2} \wedge^{\alpha}, 1^{6} \lambda^{8} \eta^{2} \eta^{\alpha}$

H

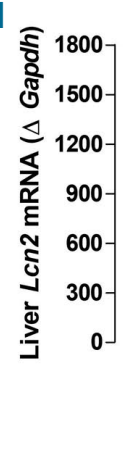

Hours after LPS injection
C

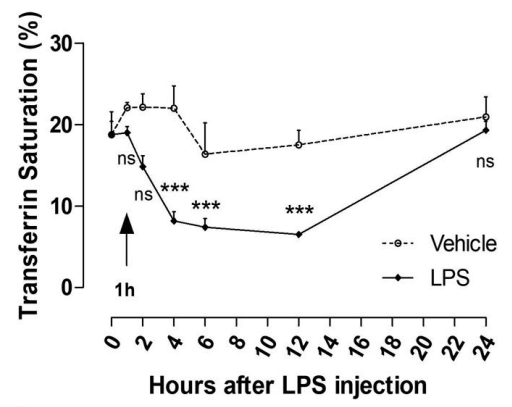

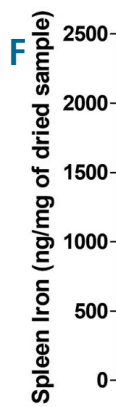

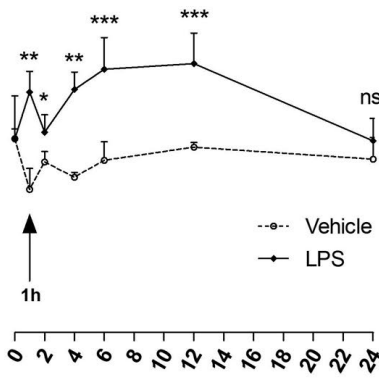

Hours after LPS injection

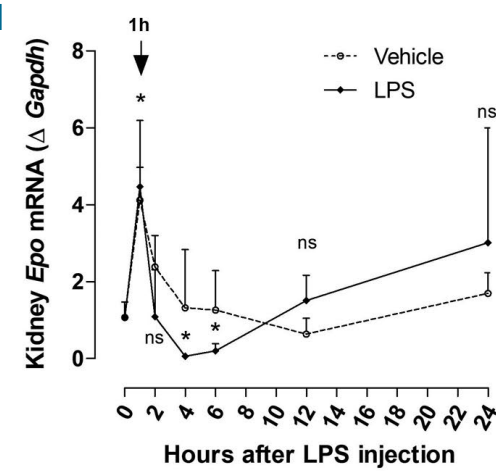

Figure 3. Effect of lipopolysaccharide (LPS) on iron homeostasis and renal Epo mRNA expression. C57BL/6J mice were injected intraperitoneally (i.p.) with saline $(0.9 \% \mathrm{NaCl}$, indicated as Vehicle) or LPS (50 $\mathrm{\mu g} / \mathrm{kg})$. Samples were collected at 0, 1, 2, 4, 6, 12, and 24 hours (h) after treatment. (A) Quantitative real-time polymerase chain reaction (qRT-PCR) for hepcidin expression in liver. (B) Serum iron and (C) serum transferrin saturation. (D and E) qRT-PCR for ferroportin (Fpn) expression in (D) liver and (E) spleen. (F) Iron content in spleen. (G) Spleen weight normalized to the body weight of the animals. (H) qRT-PCR for lipocalin (Lcn2) in liver. (I) qRT-PCR for renal Epo mRNA expression. Data are expressed as fold change $\left(2^{-\Delta \Delta C t}\right)$ relative to housekeeping genes Gapdh or Hprt. Samples were measured in duplicates (vehicle, $n=3-4$; LPS, $n=5-8$ ). Data are represented as mean \pm standard deviation. All data were analyzed for normality with Shapiro-Wilk test and equivalence of variance using Levene's test. The samples not in normal distribution were analyzed with non-parametric Kruskal-Wallis test (A and B, D-I). When the samples showed normal distribution, two-way ANOVA was performed with Bonferroni's multiple comparison test in each vehicle- or LPS-treated group compared to $0 \mathrm{~h}$ (C). ns: not significant, ${ }^{\star} P<0.05, * * P<0.01, * * * P<0.001$ compared to $0 \mathrm{~h}$.

also increased in response to LPS (Table 1). Importantly, inhibition of FGF23 signaling increased serum iron and transferrin saturation levels in both basal and LPS conditions (Figure 6A and B), and prevented LPS-induced liver and spleen iron sequestration (Figure $6 \mathrm{E}$ and $\mathrm{F}$ ). Circulating neutrophils and expression of ferritin $\mathrm{H}$ and lipocalin 2 in the liver were also significantly reduced after administration of the FGF23 C-tail blocking peptide in LPS-treated mice (Table 1 and Figure $6 \mathrm{G}$ and $\mathrm{H}$ ). However, blocking FGF23 signaling did not change the expression of liver and spleen ferroportin in response to LPS (Figure 6C and D), but in basal conditions it selectively induced splenic ferroportin mRNA and protein expression compared to controls (Figure 6D and Online Supplementary Figure S4). Taken together, these data suggest that inhibition of FGF23 signaling can alleviate hypoferremia induced by acute inflammation in mice in the absence of renal disease.

Inhibition of FGF23 signaling increases renal and EPO and EpoR

Consistent with previously published data, ${ }^{36}$ our studies show that LPS significantly downregulated renal Epo and EpoR expression after $4 \mathrm{~h}$ (Figure 7A and B). We also found that renal Hif $2 \alpha$ mRNA was significantly decreased after LPS treatment (Figure 7C), in line with the role of HIF2 as a regulator of Epo synthesis and iron metabolism. ${ }^{40}$ Importantly, inhibition of FGF23 signaling prevented the downregulation of renal Epo, EpoR, and Hif $2 \alpha$ expression caused by LPS (Figure 7A-C). We previously reported that treatment with the FGF23 C-tail blocking peptide significantly increases circulating Epo levels in mice. ${ }^{29}$ Here, we 
A

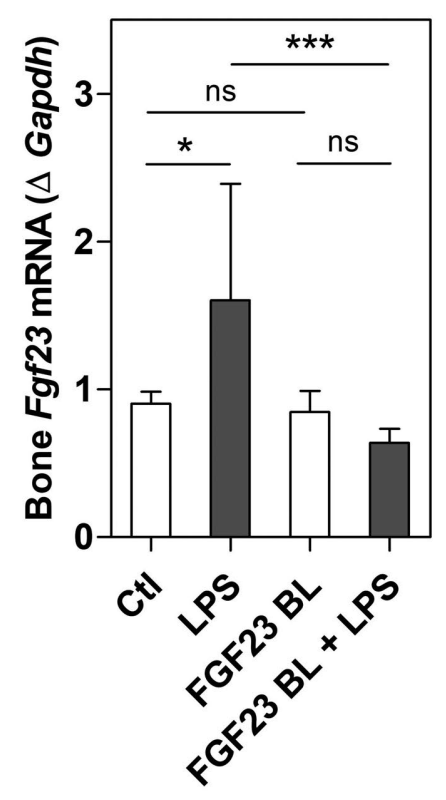

B

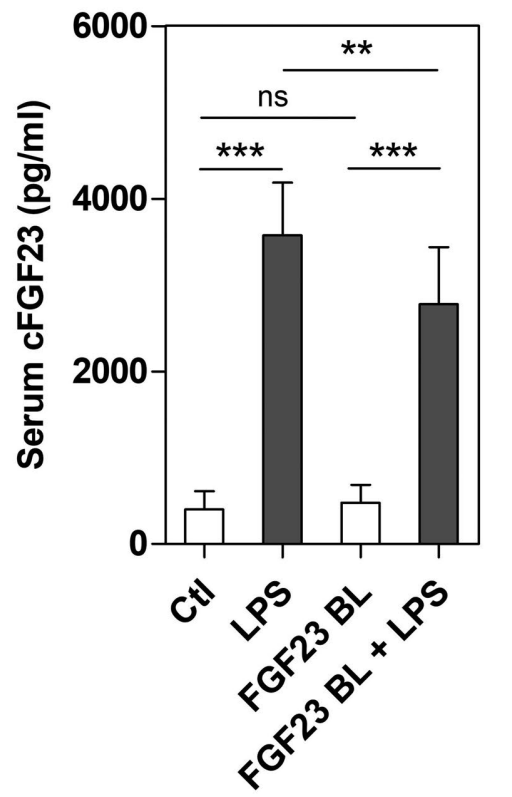

C

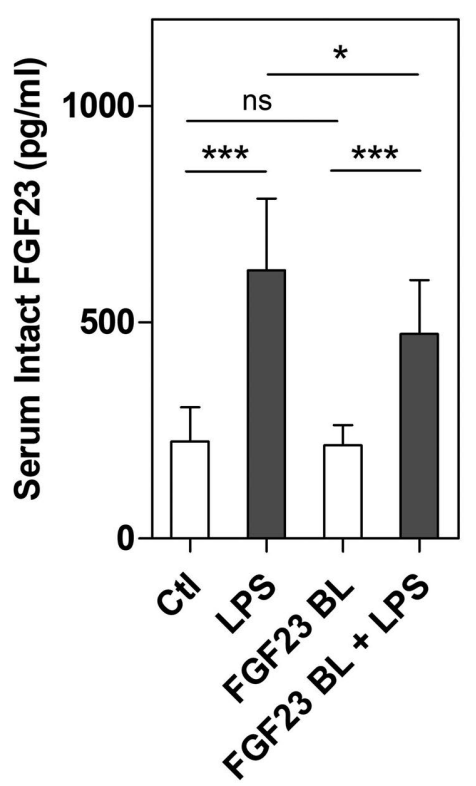

Figure 4. Inhibition of fibroblast growth factor 23 (FGF23) signaling decreases Fgf23 expression and circulating levels induced by lipopolysaccharide (LPS). C57BL/6J wild-type mice were injected intraperitoneally (i.p.) with C-tail FGF23 (1 mg/kg, indicated as FGF23 BL) or vehicle (HEPES buffer) for 8 hours (h). Mice were then challenged with LPS (i.p. $50 \mu \mathrm{g} / \mathrm{kg}$ ) or vehicle $(0.9 \% \mathrm{NaCl}$ ) for $4 \mathrm{~h}$. (A) Quantitative real-time polymerase chain reaction (qRT-PCR) for Fgf23 expression in bone. Data are expressed as fold change $\left(2^{-\Delta \Lambda C t}\right)$ relative to housekeeping gene Gapdh. (B and C) Serum concentration of (B) C-terminal FGF23 (cFGF23) and (C) intact FGF23 measured by ELISA. Samples were measured in duplicates ( $n=5-7$ per group). Data are represented as mean+standard deviation. All data were analyzed for normality with Shapiro-Wilk test and equivalence of variance using Levene's test. Because the samples did not show normal distribution, data were aligned in RANK transformation, and confirmed for normality. As the samples showed normal distribution, two-way ANOVA was performed with Bonferroni's multiple comparison test. Ctl: control (vehicle), ns: not significant, $* P<0.05, * * P<0.01$, $* * * P<0.001$.

confirmed this effect in mice injected with LPS or saline (Figure 7D) using a 10 times lower concentration of the FGF23 C-tail blocking peptide than the one we used in our previous work. ${ }^{29}$ However, we found that $4 \mathrm{~h}$ of LPS treatment at the concentration of $50 \mu \mathrm{g} / \mathrm{kg}$ were not sufficient to alter circulating Epo levels (Figure 7D). In addition to its effect on renal Epo and EpoR, LPS significantly decreased the expression of splenic and hepatic Epo and EpoR (Figure $7 \mathrm{E}-\mathrm{H})$, which was rescued by treatment with the FGF23 C-tail blocking peptide (Figure 7E-H). These data clearly demonstrate the efficiency of the C-tail FGF23 in increasing renal and extra-renal Epo and EpoR expression in the presence of inflammation, leading to increased serum Epo levels. Our findings also indicate that in the absence of inflammation, the C-tail FGF23 increases circulating Epo levels by upregulating splenic Epo expression without affecting renal or hepatic Epo expression.

\section{Discussion}

Iron is an essential micronutrient for host-pathogen interaction. During infection, pathogens develop multiple strategies to acquire iron for proliferation and virulence, such as secretion of ferric iron-binding molecules known as siderophores. ${ }^{41} \mathrm{~A}$ host defense mechanism to inhibit pathogen growth is to deprive invading pathogens of iron, a process known as nutritional immunity by sequestering iron in tissues, thus limiting availability of circulating iron and resulting in hypoferremia. ${ }^{42}$ Iron homeostasis is controlled by two proteins, the hepatic hormone hepcidin which regulates iron absorption, and the iron exporter ferroportin which exports iron into the circulation from duo- denal enterocytes, hepatocytes, and splenic reticuloendothelial macrophages that recycle the iron of senescent erythrocytes. ${ }^{43}$ Toll-like receptors (TLR) are key components of the innate immune system that recognize pathogen-associated molecular patterns (PAMP). Activation of TLR signaling plays a pivotal role in the development of the hypoferremic host response. . $3,44^{-3}$ Several toll like receptors such as TLR3, 4, 7, 7/8 and 9 have been described to induce hepcidin expression in vitro and in vivo. ${ }^{44}$

Several studies have demonstrated that iron deficiency induces FGF23 production. ${ }^{12,13,45}$ We recently reported that inhibition of FGF23 signaling using the C-tail FGF23 rescues anemia and iron deficiency in a CKD mouse model characterized by chronic inflammation, by increasing $\mathrm{EPO}$, serum iron and ferritin levels, and attenuating inflammation. ${ }^{29}$ Our goals in the present study were: (i) to decipher the kinetics of Fgf23 in response to LPS in comparison to the kinetics of inflammatory, iron metabolism, and erythropoiesis parameters; and (ii) to investigate the impact of FGF23 signaling inhibition during LPS-induced acute inflammation.

Our study showed that FGF23 is induced as early as proinflammatory cytokines in response to LPS (Figure 1), followed by upregulation of hepcidin and decrease in serum iron and transferrin saturation (Figure $3 \mathrm{~A}-\mathrm{C}$ ), suggesting that the early increase in FGF23 by LPS may contribute to hypoferremia. Previous studies have shown that FGF23 is produced by macrophages and acts locally to stimulate TNF- $\alpha$ expression via activation of FGFR $1 / \alpha-K l o t h o$ signaling. ${ }^{46}$ Moreover, acute inflammation induced by interleukin-1 $\beta$ (IL-1 $\beta$ ) or heat-killed Brucella abortus in mice significantly increased Fgf23 production. ${ }^{12}$ Thus, since FGF23 
A

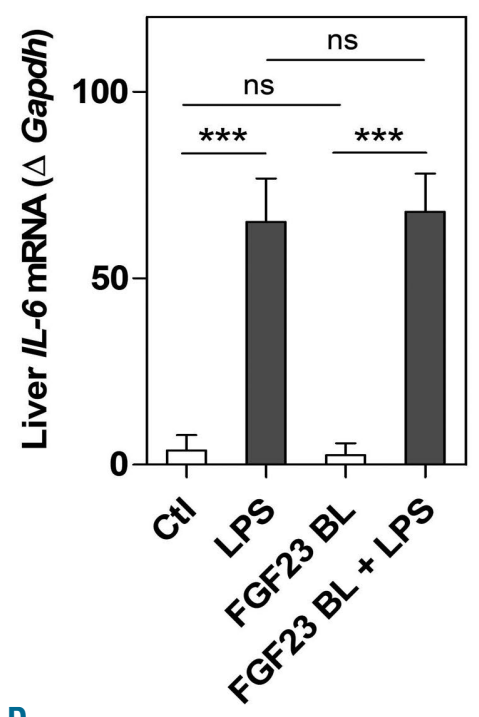

D

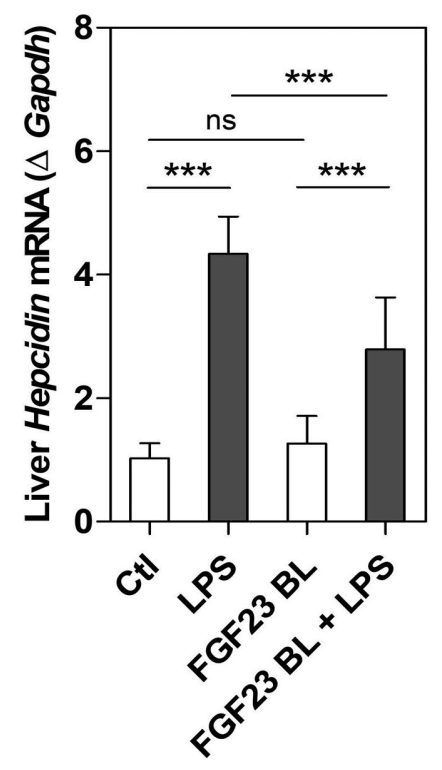

B

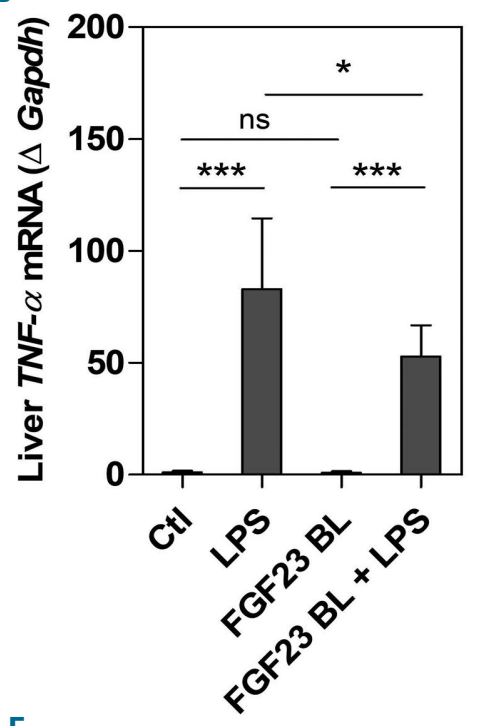

E

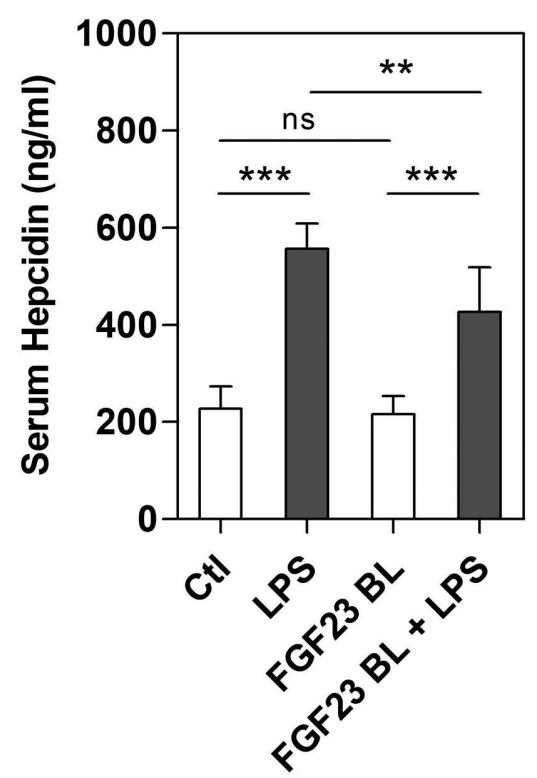

C

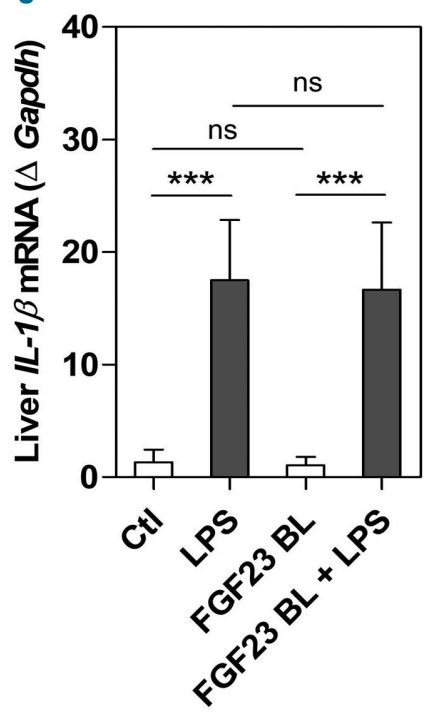

Figure 5. Effect of fibroblast growth factor 23 (FGF23) signaling disruption on inflammation and hepcidin secretion. C57BL/6J wild-type mice were treated with C-tail FGF23 (1 mg/kg, indicated as FGF23 BL) or vehicle (HEPES buffer) for 8 hours (h). Mice were then challenged with LPS (intraperitoneal $50 \mu \mathrm{g} / \mathrm{kg}$ ) or vehicle $(0.9 \% \mathrm{NaCl})$ for $4 \mathrm{~h}$. (A-D) Quantitative real-time RT-PCR for hepatic expression of $(A)$ IL-6, (B) TNF- $\alpha$, (C) IL-1 $\beta$, and (D) hepcidin. Data are expressed as fold change $\left(2^{-\Delta \Delta c t}\right)$ relative to housekeeping gene Gapdh. (E) Serum concentration of hepcidin measured by ELISA. Samples were measured in duplicates ( $n=5-7$ per group). Data are represented as mean+standard deviation. All data were analyzed for normality with Shapiro-Wilk test and equivalence of variance using Levene's test. Because the samples did not show normal distribution, data were aligned in RANK transformation, and confirmed for normality. As the samples showed normal distribution, two-way ANOVA was performed followed by Bonferroni's multiple comparison test (A-E). Ctl: control (vehicle), ns: not significant, $* P<0.05, * * P<0.01$, $* * * P<0.001$. and inflammation positively regulate each other, it is possible that one condition may precede the other.

FGF23 is a hormone primarily produced by osteocytes that affects many systems in the body. FGF23 is crucial for maintaining normal phosphate and vitamin $\mathrm{D}$ homeostasis. Its target organ for these actions is the kidney, where it inhibits phosphate reabsorption by downregulating the type II sodium-phosphate co-transporters ( $\mathrm{NaPi} 2 \mathrm{a}$ and $\mathrm{NaPi} 2 \mathrm{c})$, and reduces circulating calcitriol levels $\left(1,25(\mathrm{OH})_{2}\right.$ vitamin $\mathrm{D}_{3}$ ) by decreasing renal expression of $1-\alpha$-hydroxylase..$^{17-20,47-50}$ FGF23 levels increase significantly in CKD and are associated with kidney disease progression, greater cardiovascular risk, left ventricular hypertrophy, and mortality. ${ }^{25,51}$ Phosphate, calcium, vitamin $\mathrm{D}$, and parathyroid hormone (PTH) are known regulators of FGF23. However, in recent years, several other factors, such as inflammation, iron status, and EPO have been identified to both regulate FGF23 and be regulated by it..$^{13,29,52-58}$ Several mechanisms have been proposed to explain the association between FGF23 and inflammation. It has been shown that inflammation stimulates FGF23 either indirectly through increased hepcidin production, causing iron sequestration in macrophages and subsequent functional iron deficiency, or directly by stimulating Hif $\alpha$ transcription leading to increased HIF1 $\alpha$ which together with HIF1 $\beta$ bind to HIF response element on the Fgf23 promoter inducing its transcription. ${ }^{12}$ More recently, IL6 was reported to induce Fgf23 promoter activity through a STAT3 response element on the $F g f 23$ promoter, ${ }^{59}$ and a distal enhancer upstream of the Fgf23 transcription start site was shown to mediate inflammation-induced expression of Fgf23. ${ }^{60}$

FGF23 can directly target a number of different cell types and affect a variety of organs. Depending on the target, the mechanisms of FGF23 actions can differ in their requirement for klotho and specific FGFR isoforms, thereby activating different signaling pathways and leading to cell type- and tissue-specific effects of FGF23. ${ }^{61}$ For example, FGF23 can directly stimulate secretion of proinflammatory cytokines through activation of FGFR4 and PLC / calcineurin/NFAT signaling in the absence of klotho in the liver and bronchial epithelial cells in patients with $\mathrm{CKD}$ and chronic obstructive pulmonary disease (COPD), 
A

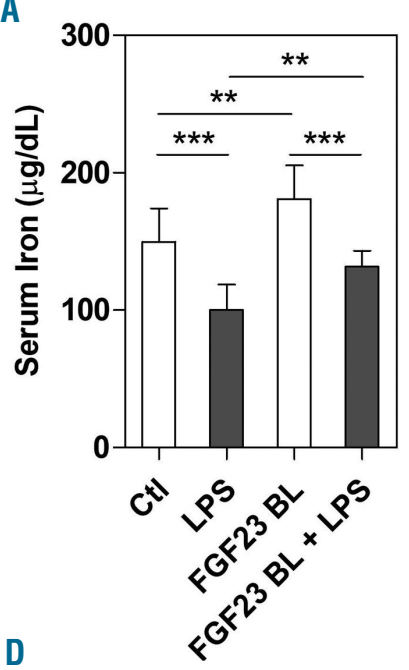

D

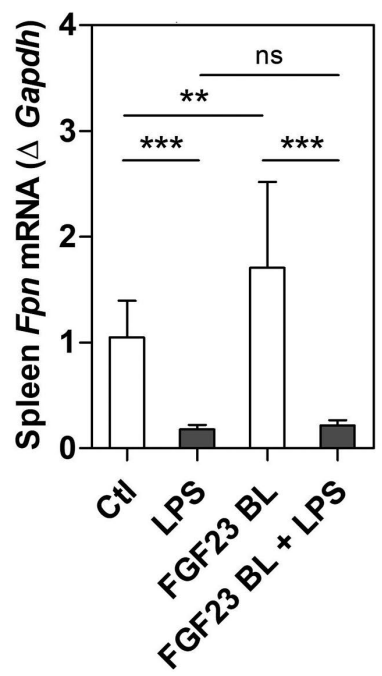

G

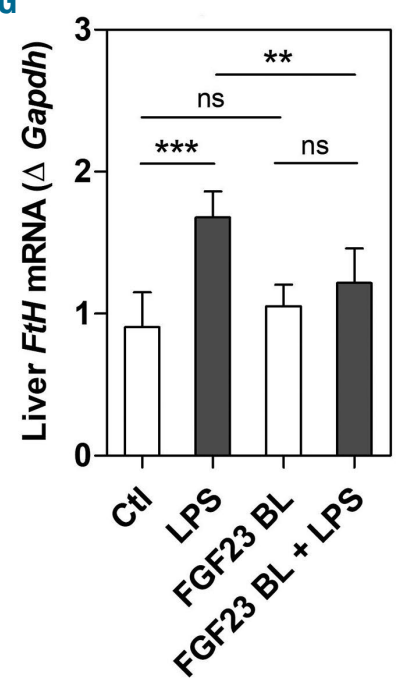

B

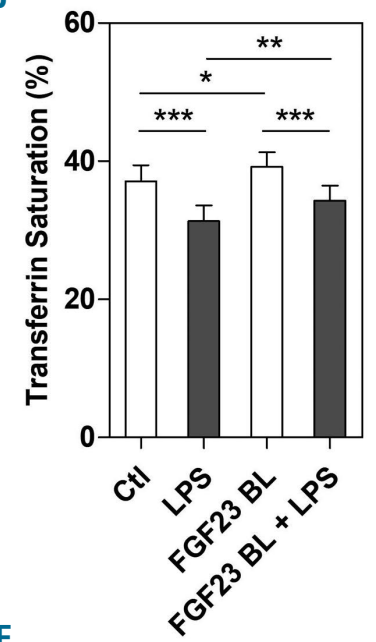

E

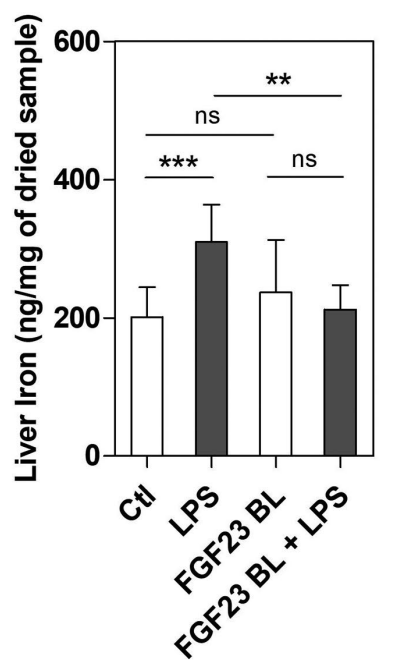

C
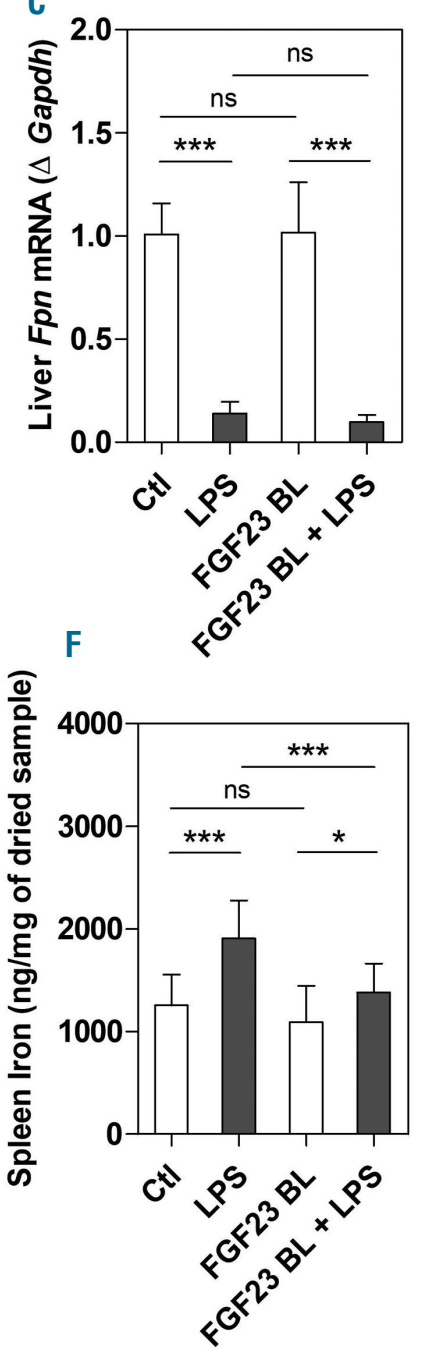

$\mathrm{H}$

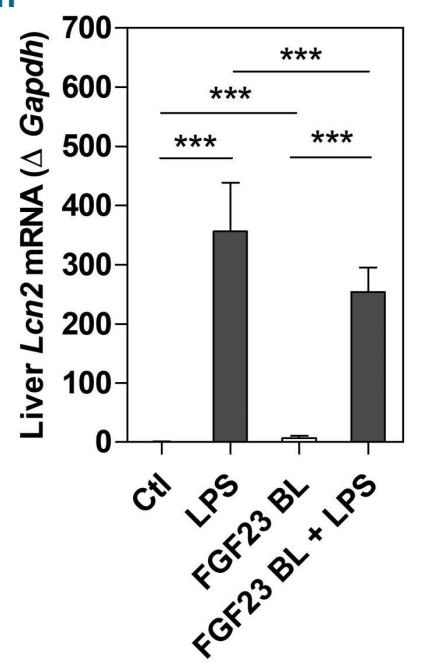

Figure 6. Inhibition of fibroblast growth factor 23 (FGF23) signaling alleviates lipopolysaccharide (LPS)-induced hypoferremia. C57BL/6J wild-type mice were treated with C-tail FGF23 (1 mg/kg, indicated as FGF23 BL) or vehicle (HEPES buffer) for 8 hours (h). Mice were then challenged with LPS (intraperitoneal 50 ug/kg) or vehicle $(0.9 \% \mathrm{NaCl})$ for $4 \mathrm{~h}$. (A) Serum iron and (B) serum transferrin saturation. (C and D) Quantitative real-time polymerase chain reaction (qRT-PCR) for ferroportin $(F p n)$ expression in (C) liver and (D) spleen. (E and F) Iron content in (E) liver and (F) spleen. (G and H) qRT-PCR for hepatic expression of (G) ferritin H and (H) lipocalin (Lcn2). Data are expressed as fold change $\left(2^{-\Delta c t}\right)$ relative to housekeeping gene Gapdh. Samples were measured in duplicates $(n=5-7$ per group). Data are represented as mean+standard deviation. All data were analyzed for normality with Shapiro-Wilk test and equivalence of variance using Levene's test. For samples with normal distribution, two-way ANOVA with Bonferroni's multiple comparison test was performed (B, E, F, and G). When the samples did not show normal distribution, they were aligned in RANK transformation, and confirmed for normality. As the samples showed normal distribution, two-way ANOVA was performed with Bonferroni's multiple comparison test (A, C, D, and H). Ctl: control (vehicle), ns: not significant, $* P<0.05, * * P<0.01, * * * P<0.001$. 
A

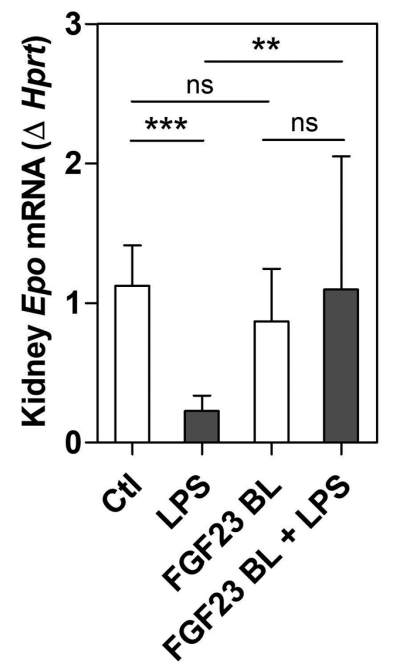

D

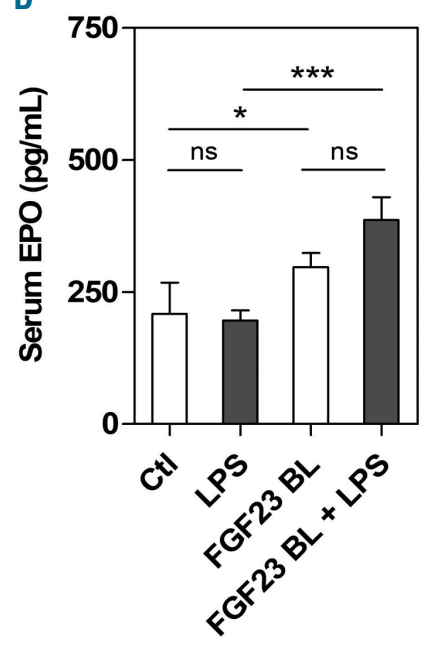

B

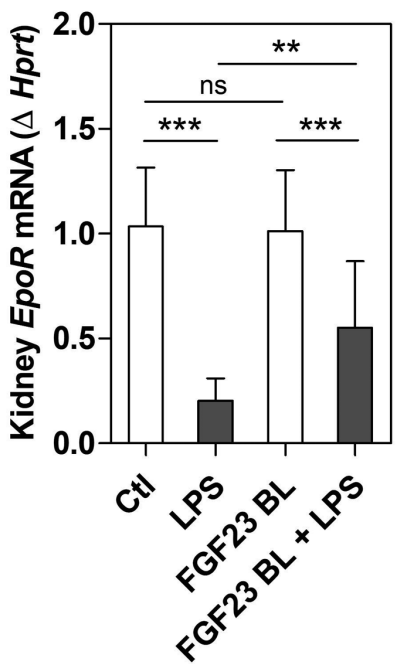

E

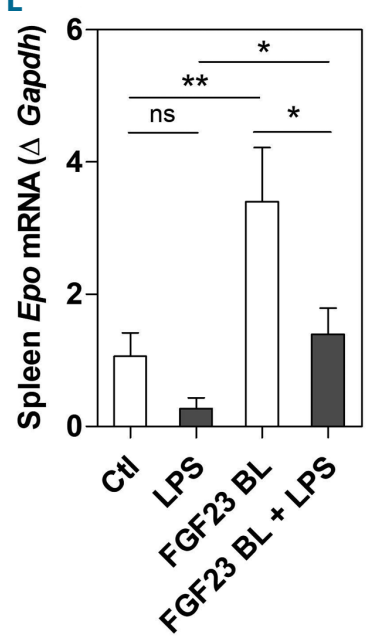

C

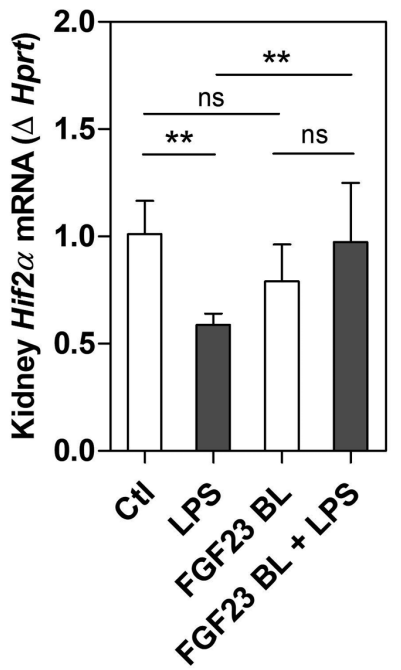

$F$

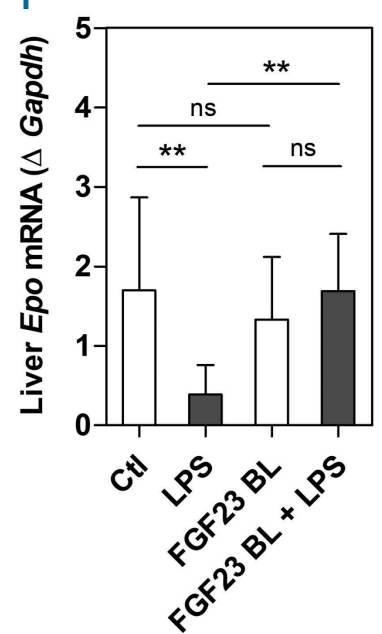

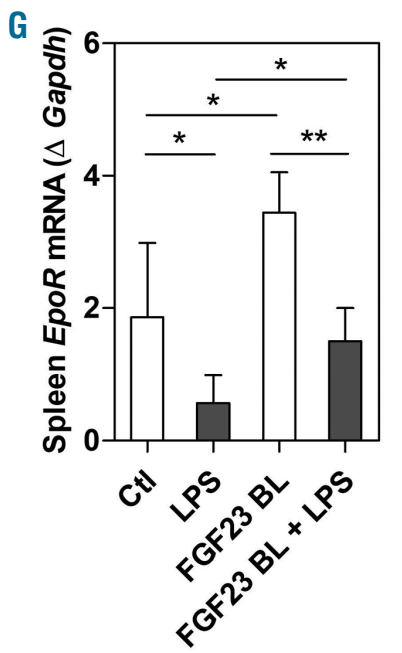

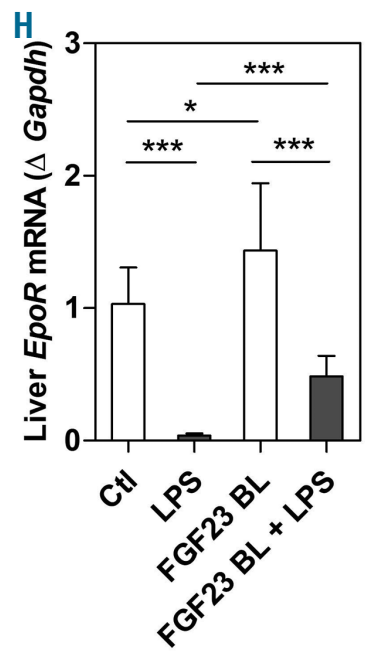

Figure 7. Inhibition of fibroblast growth factor 23 (FGF23) signaling increases renal and extra-renal EPO and EpoR mRNA expression under lipopolysaccharide (LPS)-induced hypoferremia. C57BL/6J wild-type mice were treated with C-tail FGF23 (1 mg/kg, indicated as FGF23 BL) or vehicle (HEPES buffer) for 8 hours (h). Mice were then challenged with LPS (intraperitoneal $50 \mathrm{\mu g} / \mathrm{kg}$ ) or vehicle $(0.9 \% \mathrm{NaCl})$ for $4 \mathrm{~h}$. (A-C) Quantitative real-time polymerase chain reaction (qRT-PCR) for renal expression of (A) Epo, (B) EpoR, and (C) Hif2 $\alpha$. (D) Serum concentration of Epo measured by ELISA. (E-H) qRT-PCR for Epo expression in (E) spleen and (F) liver, and EpoR expression in $(G)$ spleen and $(H)$ liver. Data are expressed as fold change $\left(2^{-\Delta \Delta c t}\right)$ relative to housekeeping genes Gapdh or Hprt. Samples were measured in duplicates ( $n=5-7$ per group). Data are represented as mean+standard deviation. All data were analyzed for normality by Shapiro-Wilk test and equivalence of variance using Levene's test. When the samples did not show normal distribution, they were aligned in RANK transformation, and confirmed for normality. As the samples showed normal distribution, two-way ANOVA was performed with Bonferroni's multiple comparison test (B, $\mathrm{F}$, and $\mathrm{H})$. The samples not in normal distribution were analyzed with non-parametric Kruskal-Wallis test (A, C-E, and G). Ctl: control (vehicle), ns: not significant, $* P<0.05, * * P<0.01, * * * P<0.001$. 
respectively. ${ }^{58,62}$ The lack of hepatic FGFR4 signaling investigation is a limitation of our study. Therefore, further experiments are required to delineate the hepatic FGFR signaling that contributes to hypoferremia. Mice deficient in Fgf23 (Fgf $\left.23^{--}\right)$exhibit aberrant bone mineralization along with high serum phosphate, calcium, and vitamin D levels, tissue and vascular calcifications, early lethality within 12 weeks after birth, ${ }^{19}$ in addition to inflammation and dysregulation of iron homeostasis (data not shown), thus limiting the possibility of using these mice to investigate the impact of FGF23 in response to hypoferremia. Here we evaluated the effect of pharmacologic inhibition of FGF23 signaling using the C-tail FGF23 in a mouse model of LPS-induced hypoferremia. Binding and cell signaling studies have shown that the C-tail of FGF23 can inhibit FGF23 signaling and antagonize its phosphaturic activity in vivo by competing with the intact FGF23 for binding to the FGFR-Klotho complex. ${ }^{27}$ Moreover, we have previously successfully used the C-tail FGF23 in a CKD mouse model to rescue renal anemia and iron deficiency and attenuate chronic inflammation. ${ }^{29}$

CKD patients, as well as mice with 5/6 nephrectomy, exhibit elevation in FGF23 levels associated with downregulation of renal Klotho expression. Our longitudinal studies showed that serum phosphate levels significantly increased the first $2 \mathrm{~h}$ of LPS treatment (Figure $2 \mathrm{~A}$ ), consistent with previous studies. ${ }^{63}$ Although the mechanism of this increase is not fully understood, studies have shown that LPS and TNF- $\alpha$ stimulate osteoclastogenesis and bone resorption, suggesting that, in conditions of systemic inflammation, there is a transcellular shift of phosphate from bone cells into the extracellular compartment, leading to hyperphosphatemia. ${ }^{63,64}$ In response to high serum phosphate levels, circulating FGF23 and Fgf23 expression significantly increased (Figure 1D-G), followed by appropriate decrease in renal Klotho and $\mathrm{NaPi2a/c}$ expression (Figure 2B-D) to increase urinary phosphate excretion (Figure 2A) in order to achieve normophosphatemia. We also investigated any potential impact of PTH on phosphate homeostasis and we found that PTH levels are 2.5fold increased $4 \mathrm{~h}$ after LPS treatment and returned to basal levels by $6 \mathrm{~h}$ after treatment in comparison to vehicle-treated mice $(0 \mathrm{~h})$ (data not shown), suggesting the possibility that the rise in serum Pi 1-2 h after LPS may contribute to PTH induction, which in turn would induce phosphaturia. However, our data exclude the possibility that PTH contributes to the increase in FGF23 because the latter reaches peak levels in circulation at the same time point as PTH. Moreover, since vitamin D is a potent stimulator of FGF23, we measured the expression of Cyp27b1, the enzyme responsible for the synthesis of the bioactive form of vitamin $\mathrm{D}$, in the kidney. We observed a 10 -fold increase in renal Cyp27b1 expression $2 \mathrm{~h}$ after LPS treatment (data not shown), suggesting that circulating levels of vitamin D would be increased. However, FGF23 levels are already significantly elevated by $2 \mathrm{~h}$ after LPS, also excluding the possibility that vitamin $\mathrm{D}$ contributes to the increase in FGF23. The present study cannot determine whether the increase in FGF23 levels in response to LPS is due to the rise of inflammatory cytokines such as IL-6, $T N F-\alpha$ and $I L-1 \beta$, the decrease in serum iron, and/or the increase in serum phosphate, all occurring after LPS treatment and prior to the increase in FGF23 levels.

In this study we also show that, although the main source of circulating FGF23 is normally the bone, in LPS- induced inflammation, organs involved in the immune system, such as the liver and spleen, significantly contribute to the increased circulating FGF23 levels (Figure 1D-G). In addition, blocking FGF23 signaling in LPS conditions resulted in significant reduction in bone (Figure 4A) and bone marrow (data not shown) Fgf23 mRNA levels, resulting in a modest but significant decrease in serum FGF23 levels (Figure 4B and C). However, spleen and liver Fgf23 expression were not affected by the cFGF23 in LPStreated animals (data not shown), suggesting that these two tissues contribute to the residual induction of circulating FGF23. This is in agreement with published data showing that spleen significantly contributes to elevated circulating FGF23 levels that rise in response to acute or chronic exposure to LPS. ${ }^{65,66}$ Moreover, mice with genetic deletion of Fgf23 $\left(F g f 23^{-/-}\right)$or its co-receptor Klotho $\left(\right.$Klotho $\left.^{-/}\right)$exhibit a reduced number of splenocytes and thymic atrophy, ${ }^{49,67}$ suggesting that Fgf 23 may play a role in the regulation of the innate and/or acquired immune system. Bone marrow Fgf23 was also significantly increased between 1-12 h after LPS treatment, possibly contributing also to the increased circulating FGF23 levels (Online Supplementary Figure S1B). $F g f 23$ production in bone was only significantly increased after $4 \mathrm{~h}$ of LPS; however, this increase correlates with a similar increase in bone Fgf23 in the saline-injected group at the same time point, suggesting that it may not be LPSdependent (Online Supplementary Figure S1A).

Moreover, in line with previously acquired knowledge that LPS-induced hypoferremia is caused through hepcidin-dependent and -independent mechanisms, ${ }^{68,69}$ our results show that treatment of mice with LPS significantly downregulates renal Epo expression (Figure 3I) following upregulation of pro-inflammatory cytokines and Fgf23 secretion. This is consistent with studies showing that inflammatory cytokines impair erythropoiesis by inhibiting production of Epo, and by directly inhibiting erythroid progenitor cell proliferation and differentiation. 35-37,69

The present study is the first to highlight the effect of FGF23 signaling on iron metabolism and its contribution to hypoferremia. Here, we show that at steady-state conditions, disruption of FGF23 signaling results in increased circulating iron levels (Figure 6A) without affecting hepcidin (Figure 5D and $\mathrm{E}$ ) or inflammatory status (Figure 5AC). This result is likely due to the rise of Epo induced by the C-tail FGF23 (Figure 7D) resulting in increased splenic Fpn mRNA and protein levels (Figure 7D and Online Supplementary Figure S4). Moreover, in response to LPS, mice treated with the C-tail FGF23 resist downregulation of Hif $2 \alpha$, a potent inducer of Epo, resulting in increased circulating EPO and Epo expression (Figure 7). Indeed, it has been reported that Epo directly stimulates intestinal iron absorption by increasing both iron uptake through upregulation of the divalent metal transporter 1 (Dmt1) and iron efflux through upregulation of the Fpn transporter. ${ }^{70}$ Studies have also shown that HIF2 $\alpha$ maintains iron balance by regulating transcription of Dmt1 and $F p n{ }^{71,72} \mathrm{Fpn}$ is rapidly induced under low iron conditions via a HIF2 $\alpha$-dependent mechanism and disruption of HIF $2 \alpha$ in the intestine inhibits the adaptive increase of intestinal Fpn during iron deficiency in mice. ${ }^{72}$ Together, the results of these studies suggest that HIF2 $\alpha$ activation may induce iron mobilization, whereas inhibition of HIF2 $\alpha$ will favor decreased iron absorption. ${ }^{71,72}$ A limitation of our study is the lack of data on the effect of cFGF23 on intestinal HIF2a, DMT1, and FPN protein levels, which 
could have provided evidence for a possible mechanism for the effect of cFGF23 on iron absorption during hypoferremia.

Our study also demonstrates that disruption of FGF23 signaling prevents LPS-induced hypoferremia by decreasing hepcidin (Figure 5D and E) and abrogating iron sequestration in liver and spleen (Figure 6). Suppression of hepcidin by cFGF23 in LPS-treated mice appears to be independent of the IL6/STAT3 pathway (Online Supplementary Figure S3). However, cFGF23 significantly decreases induction of Bmp6 transcription by LPS (Online Supplementary Figure S5), suggesting that CFGF23 may be affecting the BMP6/SMAD pathway, an important transcriptional pathway for hepcidin regulation. In support of this, our data show that splenic expression of erythroferrone (Erfe), a negative regulator of hepcidin, is decreased in mice treated with LPS, whereas mice treated with the cFGF23 under LPS conditions have significantly higher Erfe expression (Online Supplementary Figure S6). Erfe has been shown to suppress $\mathrm{BMP} / \mathrm{SMAD}$ signaling in vivo and in vitro, and to inhibit hepcidin induction by BMP6. ${ }^{73}$ Thus, our data suggest that cFGF23 decreases hepcidin induction by LPS by increasing Erfe, and possibly suppressing the BMP6/SMAD pathway.

We recently described that inhibition of FGF23 signaling using $10 \mathrm{mg} / \mathrm{kg}$ of the C-tail FGF23 rescues anemia and iron deficiency in a CKD mouse model by stimulating erythropoiesis, increasing serum iron and ferritin levels, and attenuating chronic inflammation. ${ }^{29}$ In this experimental condition, where we observed highly elevated FGF23 levels and absolute iron deficiency characterized by a decrease in circulating iron ${ }^{29}$ and iron stores (Online Supplementary Figure S7), the C-tail FGF23 significantly reduced circulating FGF23 levels and improved the impaired iron distribution by elevating circulating iron and ferritin levels ${ }^{29}$ and increasing liver iron content (Online Supplementary Figure S7). In the present study, we show that even by decreasing the concentration of the C-tail FGF23 to $1 \mathrm{mg} / \mathrm{kg}$, Epo is still induced at the steady state and after LPS (Figure 7D), and this concentration of the C-tail FGF23 is also sufficient to alleviate LPS-induced functional iron deficiency (Figure 6). However, this concentration is not sufficient to attenuate hepatic IL- 6 and $I L-1 \alpha$ induction (Figure 5A and C), but it did significantly decrease TNF- $\alpha$ expression (Figure 5B). This is consistent with other studies showing that recombinant FGF23 and increased production of endogenous FGF23, such as in Hyp mice, stimulate TNF- $\alpha$ expression in macrophages. ${ }^{46}$ Thus, inhibition of FGF23 signaling represents a therapeutic target against absolute and functional iron deficiency pathologies.

Taken together, our results show for the first time that FGF23 signaling inhibition alleviates LPS-induced acute hypoferremia.

\section{Disclosures}

No conflicts of interests to disclose.

\section{Contributions}

$R A$ developed and directed the project, performed research and data analysis, and wrote the manuscript; MYP and CLH performed research, data and statistical analyses; SJ and AG performed research; GC purified and crystallized the C-tail FGF23 and analyzed its crystal structure; $M M$ designed, refined, analyzed, and interpreted the crystal structure of the C-tail FGF23; $D S$ developed and directed the project, performed data analysis, supervised the study, and wrote the manuscript.

\section{Acknowledgments}

The authors would like to thank Anna Montagna and Elena Healy for technical assistance.

\section{Funding}

This work was supported by funds from the US Department of Defense (W81XWH-16-1-0598) to DS, and the NIH (NIDCR) to MM (DE 13686).

\section{References}

1. Gozzelino R, Arosio P. Iron homeostasis in health and disease. Int J Mol Sci. 2016;17(1).

2. Layoun A, Huang H, Calve A, Santos MM. Toll-like receptor signal adaptor protein MyD88 is required for sustained endotoxin-induced acute hypoferremic response in mice. Am J Pathol. 2012;180(6):2340-2350.

3. Schmidt PJ. Regulation of iron metabolism by hepcidin under conditions of inflammation. J Biol Chem. 2015;290(31):1897518983.

4. Ganz T. Hepcidin, a key regulator of iron metabolism and mediator of anemia of inflammation. Blood. 2003;102(3):783-788.

5. Kemna E, Pickkers P, Nemeth E, van der Hoeven H, Swinkels D. Time-course analysis of hepcidin, serum iron, and plasma cytokine levels in humans injected with LPS. Blood. 2005;106(5):1864-1866.

6. Nemeth E, Valore EV, Territo M, Schiller G, Lichtenstein A, Ganz T. Hepcidin, a putative mediator of anemia of inflammation, is a type II acute-phase protein. Blood. 2003;101(7):2461-2463.

7. Nemeth E, Tuttle MS, Powelson J, et al. Hepcidin regulates cellular iron efflux by binding to ferroportin and inducing its internalization. Science. 2004;306(5704):
2090-2093

8. Ganz T, Nemeth E. Iron homeostasis in host defence and inflammation. Nat Rev Immunol. 2015;15(8):500-510.

9. Weiss G. Iron metabolism in the anemia of chronic disease. Biochim Biophys Acta. 2009;1790(7):682-693.

10. Babitt JL, Lin HY. Mechanisms of anemia in CKD. J Am Soc Nephrol. 2012;23(10):16311634.

11. Wolf M. Update on fibroblast growth factor 23 in chronic kidney disease. Kidney Int. 2012;82(7):737-747.

12. David V, Martin A, Isakova T, Spaulding C, Qi L, Ramirez V, et al. Inflammation and functional iron deficiency regulate fibroblast growth factor 23 production. Kidney Int. 2016;89(1):135-146.

13. Farrow EG, Yu X, Summers LJ, et al. Iron deficiency drives an autosomal dominant hypophosphatemic rickets (ADHR) phenotype in fibroblast growth factor-23 (Fgf23) knock-in mice. Proc Natl Acad Sci U S A. 2011;108(46):E1146-55.

14. White KE, Carn G, Lorenz-Depiereux B, Benet-Pages A, Strom TM, Econs MJ. Autosomal-dominant hypophosphatemic rickets (ADHR) mutations stabilize FGF-23. Kidney Int. 2001;60(6):2079-2086.

15. Shimada T, Mizutani S, Muto $\mathrm{T}$, et al. Cloning and characterization of FGF23 as a causative factor of tumor-induced osteomalacia. Proc Natl Acad Sci U S A. 2001; 98(11):6500-6505.

16. Perwad F, Zhang MY, Tenenhouse HS Portale AA. Fibroblast growth factor 23 impairs phosphorus and vitamin D metabolism in vivo and suppresses 25 -hydroxyvitamin D-1alpha-hydroxylase expression in vitro. Am J Physiol Renal Physiol. 2007;293(5):F1577-1583.

17. Shimada T, Hasegawa H, Yamazaki Y, et al. FGF-23 is a potent regulator of vitamin D metabolism and phosphate homeostasis. J Bone Miner Res. 2004;19(3):429-435.

18. Sitara D, Kim S, Razzaque MS, et al. Genetic evidence of serum phosphateindependent functions of FGF-23 on bone. PLoS Genet. 2008;4(8):e1000154

19. Sitara D, Razzaque MS, Hesse M, et al. Homozygous ablation of fibroblast growth factor-23 results in hyperphosphatemia and impaired skeletogenesis, and reverses hypophosphatemia in Phex-deficient mice. Matrix Biol. 2004;23(7):421-432.

20. Sitara D, Razzaque MS, St-Arnaud R, et al. Genetic ablation of vitamin $\mathrm{D}$ activation pathway reverses biochemical and skeletal anomalies in Fgf-23-null animals. Am J Pathol. 2006;169(6):2161-2170.

21. Eicher EM, Southard JL, Scriver CR, Glorieux FH. Hypophosphatemia: mouse 
model for human familial hypophosphatemic (vitamin D-resistant) rickets. Proc Natl Acad Sci U S A. 1976;73(12):4667-4671.

22. Loffing J, Lotscher M, Kaissling B, et al. Renal $\mathrm{Na} / \mathrm{H}$ exchanger NHE-3 and $\mathrm{Na}-\mathrm{PO} 4$ cotransporter $\mathrm{NaPi}-2$ protein expression in glucocorticoid excess and deficient states. J Am Soc Nephrol. 1998;9(9):1560-1567.

23. Strom TM, Francis F, Lorenz B, et al. Pex gene deletions in Gy and Hyp mice provide mouse models for X-linked hypophosphatemia. Hum Mol Genet. 1997;6(2):165171

24. Martin A, David V, Quarles LD. Regulation and function of the FGF23/klotho endocrine pathways. Physiol Rev. 2012;92(1):131-155.

25. Faul C, Amaral AP, Oskouei B, et al. FGF23 induces left ventricular hypertrophy. J Clin Invest. 2011;121(11):4393-4408.

26. Bacic D, Capuano P, Baum M, et al. Activation of dopamine D1-like receptors induces acute internalization of the renal $\mathrm{Na}+$ /phosphate cotransporter NaPi-Ila in mouse kidney and OK cells. Am J Physiol Renal Physiol. 2005;288(4):F740-F747.

27. Goetz R, Nakada Y, Hu MC, et al. Isolated C-terminal tail of FGF23 alleviates hypophosphatemia by inhibiting FGF23FGFR-Klotho complex formation. Proc Natl Acad Sci U S A. 2010;107(1):407-412.

28. Moe OW, Tejedor A, Levi M, Seldin DW, Preisig PA, Alpern RJ. Dietary $\mathrm{NaCl}$ modulates $\mathrm{Na}(+)-\mathrm{H}+$ antiporter activity in renal cortical apical membrane vesicles. Am J Physiol. 1991;260(1 Pt 2):F130-137.

29. Agoro R, Montagna A, Goetz R, et al. Inhibition of fibroblast growth factor 23 (FGF23) signaling rescues renal anemia. FASEB J. 2018;32(7):3752-3764

30. Agoro R, Taleb M, Quesniaux VFJ, Mura C. Cell iron status influences macrophage polarization. PLoS One. 2018; 13(5):e0196921

31. Barry M, Sherlock S. Measurement of liveriron concentration in needle-biopsy specimens. Lancet. 1971;1(7690):100-103

32. Pigeon C, Ilyin G, Courselaud B, et al. A new mouse liver-specific gene, encoding a protein homologous to human antimicrobial peptide hepcidin, is overexpressed during iron overload. J Biol Chem. 2001;276(11):7811-7819.

33. Rishi G, Wallace DF, Subramaniam VN. Hepcidin: regulation of the master iron regulator. Biosci Rep. 2015;35(3).

34. Flo TH, Smith KD, Sato S, et al. Lipocalin 2 mediates an innate immune response to bacterial infection by sequestrating iron. Nature. 2004;432(7019):917-21.

35. Faquin WC, Schneider TJ, Goldberg MA. Effect of inflammatory cytokines on hypoxia-induced erythropoietin production. Blood. 1992:79(8):1987-1994.

36. Frede S, Fandrey J, Pagel H, Hellwig T, Jelkmann W. Erythropoietin gene expression is suppressed after lipopolysaccharide or interleukin-1 beta injections in rats. Am J Physiol. 1997;273(3 Pt 2):R1067-1071.

37. Vannucchi AM, Grossi A, Rafanelli D, Statello M, Cinotti S, Rossi-Ferrini P. Inhibition of erythropoietin production in vitro by human interferon gamma. $\mathrm{Br} \mathrm{J}$ Haematol. 1994;87(1):18-23.

38. Goetz R, Beenken A, Ibrahimi OA, et al. Molecular insights into the klotho-dependent, endocrine mode of action of fibroblast growth factor 19 subfamily members. Mol Cell Biol. 2007:27(9):3417-3428.

39. Rivera S, Nemeth E, Gabayan V, Lopez MA, Farshidi D, Ganz T. Synthetic hep- cidin causes rapid dose-dependent hypoferremia and is concentrated in ferroportincontaining organs. Blood. 2005;106(6): 2196-2199.

40. Haase VH. Regulation of erythropoiesis by hypoxia-inducible factors. Blood Rev. 2013; 27(1):41-53.

41. Ward RJ, Crichton RR, Taylor DL, Della Corte L, Srai SK, Dexter DT. Iron and the immune system. J Neural Transm (Vienna). 2011;118(3):315-328.

42. Hennigar SR, McClung JP. Nutritional immunity: starving pathogens of trace minerals. Am J Lifestyle Med. 2016;10(3):170 173.

43. Ganz T. Hepcidin and iron regulation, 10 years later. Blood. 2011;117(17):4425-4433.

44. Agoro R, Mura C. Inflammation-induced up-regulation of hepcidin and down-regulation of ferroportin transcription are dependent on macrophage polarization. Blood Cells, Molecules and Diseases. 2016;61:16-25.

45. Hanudel MR, Laster M, Salusky IB. Nonrenal-related mechanisms of FGF23 pathophysiology. Curr Osteoporos Rep. 2018:16(6):724-729

46. Han X, Li L, Yang J, King G, Xiao Z, Quarles $\mathrm{LD}$. Counter-regulatory paracrine actions of FGF-23 and 1,25(OH)2 D in macrophages. FEBS Lett. 2016;590(1):53-67.

47. Babitt JL, Sitara D. Crosstalk between fibroblast growth factor 23, iron, erythropoietin, and inflammation in kidney disease. Curr Opin Nephrol Hypertens. 2019:28(4):304-310

48. Gattineni J, Bates C, Twombley K, et al. FGF23 decreases renal NaPi-2a and NaPi-2c expression and induces hypophosphatemia in vivo predominantly via FGF receptor 1 Am J Physiol Renal Physiol. 2009. 297(2):F282-F291

49. Razzaque MS, Sitara D, Taguchi T, StArnaud R, Lanske B. Premature aging-like phenotype in fibroblast growth factor 23 null mice is a vitamin $\mathrm{D}$-mediated process. FASEB J. 2006;20(6):720-722.

50. Shimada T, Kakitani M, Yamazaki Y, et al. Targeted ablation of Fgf23 demonstrates an essential physiological role of FGF23 in phosphate and vitamin D metabolism. J Clin Invest. 2004;113(4):561-568

51. Leaf DE, Siew ED, Eisenga MF, et al Fibroblast growth factor 23 associates with death in critically ill patients. Clin J Am Soc Nephrol. 2018;13(4):531-541

52. Clinkenbeard EL, Hanudel MR, Stayrook $\mathrm{KR}$, et al. Erythropoietin stimulates murine and human fibroblast growth factor-23, revealing novel roles for bone and bone marrow. Haematologica. 2017; 102(11):e427-e430

53. Coe LM, Madathil SV, Casu C, Lanske B, Rivella S, Sitara D. FGF-23 is a negative regulator of prenatal and postnatal erythropoiesis. J Biol Chem. 2014;289(14):97959810

54. Daryadel A, Bettoni C, Haider T, et al. Erythropoietin stimulates fibroblast growth factor 23 (FGF23) in mice and men. Pflugers Arch. 2018;470(10):1569-1582

55. Hanudel MR, Eisenga MF, Rappaport M, et al. Effects of erythropoietin on fibroblast growth factor 23 in mice and humans. Nephrol Dial Transplant. 2019;34(12):20572065

56. Imel EA, Peacock M, Gray AK, Padgett LR, Hui SL, Econs MJ. Iron modifies plasma FGF23 differently in autosomal dominant hypophosphatemic rickets and healthy humans. J Clin Endocrinol Metab 2011;96(11):3541-3549.

57. Nam KH, Kim H, An SY, et al. Circulating fibroblast growth factor-23 levels are associated with an increased risk of anemia development in patients with nondialysis chronic kidney disease. Sci Rep. 2018;8 (1):7294.

58. Singh S, Grabner A, Yanucil C, et al Fibroblast growth factor 23 directly targets hepatocytes to promote inflammation in chronic kidney disease. Kidney Int 2016;90(5):985-996.

59. Durlacher-Betzer K, Hassan A, Levi R, Axelrod J, Silver J, Naveh-Many T. Interleukin-6 contributes to the increase in fibroblast growth factor 23 expression in acute and chronic kidney disease. Kidney Int. 2018:94(2):315-325.

60. Onal M, Carlson AH, Thostenson JD, et al. A novel distal enhancer mediates inflammation-, PTH-, and early onset murine kidney disease-induced expression of the mouse Fgf23 gene. JBMR Plus. 2018;2(1):32-47.

61. Richter B, Faul C. FGF23 actions on target tissues-with and without Klotho. Front Endocrinol (Lausanne). 2018;9:189.

62. Krick S, Grabner A, Baumlin N, et al Fibroblast growth factor 23 and Klotho contribute to airway inflammation. Eur Respir J. 2018;52(1)

63. Ikeda S, Yamamoto $H$, Masuda M, et al. Downregulation of renal type IIa sodiumdependent phosphate cotransporter during lipopolysaccharide-induced acute inflammation. Am J Physiol Renal Physiol. 2014:306(7):F744-F750

64. Abu-Amer Y, Ross FP, Edwards J Teitelbaum SL. Lipopolysaccharide-stimulated osteoclastogenesis is mediated by tumor necrosis factor via its P55 receptor. J Clin Invest. 1997;100(6):1557-1565.

65. Bansal S, Friedrichs WE, Velagapudi C, et al Spleen contributes significantly to increased circulating levels of fibroblast growth factor 23 in response to lipopolysaccharideinduced inflammation. Nephrol Dial Transplant. 2017;32(6):960-968.

66. Masuda Y, Ohta H, Morita Y, et al Expression of Fgf23 in activated dendritic cells and macrophages in response to immunological stimuli in mice. Biol Pharm Bull. 2015;38(5):687-693

67. Okada S, Yoshida T, Hong Z, et al Impairment of $\mathrm{B}$ lymphopoiesis in precocious aging (klotho) mice. Int Immunol 2000;12(6):861-871.

68. Deschemin IC, Vaulont S. Role of hepcidin in the setting of hypoferremia during acute inflammation. PLoS One. 2013;8(4):e61050.

69. Wang CY, Babitt JL. Hepcidin regulation in the anemia of inflammation. Curr Opin Hematol. 2016;23(3):189-197.

70. Srai SK, Chung B, Marks J, et al Erythropoietin regulates intestinal iron absorption in a rat model of chronic renal failure. Kidney Int. 2010;78(7):660-667.

71. Mastrogiannaki M, Matak P, Keith B Simon MC, Vaulont S, Peyssonnaux C HIF-2alpha, but not HIF-1alpha, promotes iron absorption in mice. J Clin Invest. 2009;119(5):1159-1166.

72. Taylor M, Qu A, Anderson ER, et al. Hypoxia-inducible factor-2alpha mediate the adaptive increase of intestinal ferroportin during iron deficiency in mice. Gastroenterology. 2011;140(7):2044-2055

73. Arezes J, Foy N, McHugh K, et al. Erythroferrone inhibits the induction of hepcidin by BMP6. Blood. 2018;132(14): 1473-1477. 